\title{
Network Pharmacology- and Molecular Docking-Based Identification of Potential Phytocompounds from Argyreia capitiformis in the Treatment of Inflammation
}

\author{
Ahmad J. Obaidullah (D), ${ }^{1}$ Mohammed M. Alanazi $\left(\mathbb{D},{ }^{1}\right.$ Nawaf A. Alsaif, ${ }^{1}$ Ashwag S. Alanazi, \\ Hussam Albassam $₫,{ }^{3}$ Alanazi AZ $₫,{ }^{3}$ Osama I. Alwassil $\odot{ }^{4}$ \\ Ali M. Alqahtani $\mathbb{D}^{5}{ }^{5}$ and Abu Montakim Tareq ${ }^{6}$ \\ ${ }^{1}$ Department of Pharmaceutical Chemistry, College of Pharmacy, King Saud University, P.O. Box 2457, \\ Riyadh 11451, Saudi Arabia \\ ${ }^{2}$ Department of Pharmaceutical Sciences, College of Pharmacy, Princess Nourah Bint Abdulrahman University, \\ Riyadh 84428, Saudi Arabia \\ ${ }^{3}$ Department of Pharmacology and Toxicology, College of Pharmacy, King Saud University, P.O. Box 2457, \\ Riyadh 11451, Saudi Arabia \\ ${ }^{4}$ Department of Pharmaceutical Sciences, College of Pharmacy, King Saud bin Abdulaziz University for Health Sciences, \\ Riyadh 11481, Saudi Arabia \\ ${ }^{5}$ Department of Pharmacology, College of Pharmacy, King Khalid University, Abha 62529, Saudi Arabia \\ ${ }^{6}$ Department of Pharmacy, International Islamic University Chittagong, Chittagong 4318, Bangladesh
}

Correspondence should be addressed to Ahmad J. Obaidullah; aobaidullah@ksu.edu.sa

Received 18 December 2021; Revised 3 January 2022; Accepted 15 January 2022; Published 31 January 2022

Academic Editor: Ruchika Garg

Copyright (C) 2022 Ahmad J. Obaidullah et al. This is an open access article distributed under the Creative Commons Attribution License, which permits unrestricted use, distribution, and reproduction in any medium, provided the original work is properly cited.

\begin{abstract}
The methanolic extract of Argyreia capitiformis stem was examined for anti-inflammatory activities following network pharmacology analysis and molecular docking study. Based on gas chromatography-mass spectrometry (GC-MS) analysis, 49 compounds were identified from the methanolic extract of A. capitiformis stem. A network pharmacology analysis was conducted against the identified compounds, and Kyoto Encyclopedia of Genes and Genomes (KEGG) pathway analysis and Gene Ontology analysis of biological processes and molecular functions were performed. Six proteins (IL1R1, IRAK4, MYD88, TIRAP, TLR4, and TRAF6) were identified from the KEGG pathway analysis and subjected to molecular docking study. Additionally, six best ligand efficiency compounds and positive control (aspirin) from each protein were evaluated for their stability using the molecular dynamics simulation study. Our study suggested that IL1R1, IRAK4, MYD88, TIRAP, TLR4, and TRAF6 proteins may be targeted by compounds in the methanolic extract of A. capitiformis stem to provide anti-inflammatory effects.
\end{abstract}

\section{Introduction}

Inflammation describes a biological process that occurs in tissues to protect the host against harmful stimuli, such as microorganisms and abnormal or damaged cells. Inflammation stimulates the immune system and regulates protective responses via immune cells, blood vessels, and molecular biological agents $[1,2]$. Many chronic diseases, including cardiovascular and gastrointestinal illnesses, diabetes, rheumatism, and cancer, are associated with upregulated inflammation [3]. Chronic diseases represent a major human health concern according to the World Health Organization (WHO). The incidence of chronic inflammation-related disorders is expected to steadily increase in the United States (US) over the next 30 years. Approximately 125 million people in the US were diagnosed with chronic diseases in 2000, with 61 million (21\%) having multiple conditions [4-6]. Typically, cellular and molecular 
mechanisms and interactions among various factors can efficiently limit the potential damage and prevent further infection during an acute inflammatory response, resulting in the eventual repair of cellular homeostasis following the resolution of acute inflammation. However, uncontrolled acute inflammation can develop into chronic inflammation, causing a range of chronic inflammatory diseases [7]. Three out of five people worldwide die from chronic inflammatory conditions, such as stroke, respiratory infections, cardiovascular diseases, cancers, diabetes, and obesity [4-6]. A growing interest in the use of medicinal plants has developed for the treatment and management of diseases in an effort to identify safer and more efficient anti-inflammatory agents for the prevention of inflammatory conditions rather than using synthetic anti-inflammatory drugs [8].

Argyreia capitiformis (Poir.) Ooststr. is a member of the Convolvulaceae family of the Argyreia genus, which is not toxic and has medicinal and ornamental uses $[9,10]$. Traditionally, a paste made from the leaves of A. capitiformis has been used as an effective treatment of bruising on the legs. A. capitiformis has also been used in traditional medicine as a purgative and to treat sexual debility and ear pain [10-12]. Several studies have been reported for the Argyreia species, including antioxidant, anti-inflammatory, immunomodulatory, and CNS activities with several bioactive compounds [13-15]. However, no such study has been evaluated for A. capitiformis except the recent study on the methanolic extract of A. capitiformis leaves that suppressed the nuclear factorkappa B (NF- $\kappa \mathrm{B})$ pathway and inhibited the lipopolysaccharide-induced production of nitric oxide and inducible nitric oxide synthase in RAW 264.7 cells, demonstrating anti-inflammatory activities [9]. However, the chemical compounds found in A. capitiformis that are responsible for these anti-inflammatory effects and the underlying mechanisms remain unknown. Further research is necessary to identify the potential lead compounds responsible for these biological anti-inflammatory outcomes.

Network pharmacology has become a widely accessible analysis method following the increased availability of biomedical data sets during the postgenomic period, supporting the growth of the fields of systems biology and polypharmacology [16]. Complex compound-gene and compound-protein interactions can be evaluated systematically to develop a prototype for efficient therapy. New therapeutic mechanisms may be discovered by network pharmacology analysis, which is oriented toward a "multigoals, multi-disease" paradigm rather than "one target, one drug" [17-19]. Network pharmacology represents an effective method for selecting and elucidating the synergistic effects among bioactive chemicals through the mechanistic exploration of effects on multiple disease pathways $[19,20]$. Additionally, spectrometric and chromatographic technologies used in the initial evaluation of medicinal plants provide valuable information on bioactive activities that aid in the selection of biologically active species. Alkaloids, phenolic compounds, organic acids, esters, and amino acids are among the chemicals that GC-MS can detect quickly and accurately. Thus, in this investigation, GC-MS was used to detect and identify phytochemical constituents in A. capitiformis [21-24].

The network pharmacology approach connects targeted genes with the effects of bioactive compounds; thus, the present study was designed to elucidate the anti-inflammatory effects of the methanolic extract of A. capitiformis stem using a network pharmacology approach. Bioactive compounds in the methanolic extract of $A$. capitiformis stem were identified for this study using gas chromatographymass spectrometry (GC-MS) analysis, followed by a molecular docking assay to investigate potential ligand-receptor interactions, including the assessment of binding affinity and stability.

\section{Materials and Methods}

2.1. Plant Extraction. The stems of A. capitiformis were collected from the Sitakunda Eco-park, Chittagong, Bangladesh, in March 2020 and later identified by a taxonomist. The stems were subjected to air-drying and ground to a coarse powder. The powder $(200 \mathrm{~g})$ was soaked in methanol (1 L) for 7 days $[25,26]$. Subsequently, the extract was filtered through Whatman filter paper and evaporated at $45^{\circ} \mathrm{C}$. After the evaporation, $2.67 \mathrm{~g}$ of the black methanol extract yield $(1.34 \%)$ was collected in an amber glass vial and refrigerated at $4^{\circ} \mathrm{C}$ until further use.

2.2. GC-MS Analysis. An Agilent GC 7890A (Agilent Technologies Inc., Wilmington, DE, USA), combined with a triple-axis detector $5975 \mathrm{C}$ single-quadrupole mass spectrometer, was used for GC-MS analysis. The chromatographic column was an Agilent HP 5MS column ( $30 \mathrm{~m} \times 0.25 \mathrm{~mm} \times 0.25 \mu \mathrm{m}$ film thickness), using high-purity helium as the gas carrier at a flow rate of $1 \mathrm{~mL}$ per min. The injector temperature was $230^{\circ} \mathrm{C}$, and the sample was injected using a splitless injector at $20: 1$. The temperature was set primarily to $40^{\circ} \mathrm{C}$ (held for $1 \mathrm{~min}$ ), raised to $150^{\circ} \mathrm{C}$ at a rate of $5^{\circ} \mathrm{C}$ per min (held for $2 \mathrm{~min}$ ), before being increased to $300^{\circ} \mathrm{C}$ at a rate of $5^{\circ} \mathrm{C}$ per min (held for $10 \mathrm{~min}$ ). The temperature of the MS ion source was set to $150^{\circ} \mathrm{C}$, and the temperature of the inlet line was set to $280^{\circ} \mathrm{C}$. The scan range was set between 50 and 550 mass, with $70 \mathrm{eV}$ electron energy and a 4-min solvent delay. Finally, by comparing the spectra against the NIST 2008 database (National Institute of Standard and Technology library), tentative compounds were identified. The total analysis time required for the sample was $65 \mathrm{~min}$ [27].

2.3. Network Pharmacology. The network pharmacology analysis was performed using the STITCH platform (http:// stitch.embl.de/) to identify putative associations between the identified compounds and target genes. Multiple compound targets were identified using the Homo sapiens genome [27-29]. Multiple functional nodes and edges were identified in the network. Kyoto Encyclopedia of Genes and Genomes (KEGG) analysis was performed on the components identified in the network to obtain a biological interpretation of 
the vast list of potential targets and to identify potential antiinflammatory pathways that are targeted.

The STRING (search tool for retrieval of interacting genes) database (https://string-db.org), which includes predicted protein-protein interactions (PPIs), was used to predict functional protein interactions [30].

\subsection{Molecular Docking Study}

2.4.1. Protein Selection and Preparation. Six targeted proteins were selected from the KEGG analysis: interleukin 1 (IL-1) receptor type 1 (IL1R1; PDB: 1ITB) [31], IL-1 receptorassociated kinase 4 (IRAK4; PDB: 6EGA) [32], myeloid differentiation factor 88 (MYD88; PDB: 4EO7) [33], TIR domain-containing adaptor protein (TIRAP; PDB: 4FZ5) [34], Toll-like receptor 4 (TLR4; PDB: 3FXI) [35], and tumor necrosis factor (TNF) receptor-associated factor 6 (TRAF6; PDB: 3HCT) [36]. Protein structures were retrieved in .pdb format from the Protein Data Bank (http://www.rcsb.org/ pdb). The proteins were prepared using Schrödinger (Maestro v11.1), utilizing the force field OPLS3 [27].

2.4.2. Ligand Preparation. We selected 47 compounds identified from $A$. capitiformis, according to the qualitative GC-MS analysis, which we submitted to the molecular docking study. The selected compounds were retrieved in .$s d f$ format from the PubChem database. In addition, aspirin (CID: 2244) was utilized in this study as a positive antiinflammatory control. The three-dimensional (3D) structures of the selected compounds were constructed in Schrödinger using LigPrep (Maestro v11.1), utilizing the force field OPLS3.

2.4.3. Grid Generation and Molecular Docking. To create receptor grids and execute a molecular docking analysis, Glide (Schrödinger, Maestro v11.1) was used. The grids were generated in Glide with the default settings and the OPLS3 force field. A cubic box with a boundary box $(14 \AA \times 14 \AA \times 14 \AA)$ was specified for the receptors. All docking studies were conducted using Glide's standard precision (SP) and flexible docking modes, and the lowest docking score for each ligand was recorded.

2.4.4. MM-GBSA and Ligand Efficiency Analysis. The free energies of binding $(\Delta G ; \mathrm{kcal} / \mathrm{mol})$ for each ligand and the target receptors were calculated using the Schrödinger software package Prime/MM-GBSA module (OPLS3) $[37,38]$. The ligand efficiency (LE) was assessed for each ligand by obtaining the ratio of $\Delta G$ to the number of heavy atoms (NHA): $\mathrm{LE}=-(\Delta G) / \mathrm{NHA}[39]$.

2.5. Molecular Dynamics Simulation. The molecular dynamics simulation study was conducted in YASARA dynamics by the aid of AMBER14 force field [40, 41]. The docked complexes were optimized and cleaned, and hydrogen bond network system was oriented. The cubic simulation cell was created where the TIP3P solvation model was used with periodic boundary conditions [42]. The simulation system was neutralized at $310 \mathrm{~K}$ temperature, $\mathrm{pH}$ 7.4 , and $0.9 \% \mathrm{NaCl}$. The initial energy minimization was conducted by steepest grained algorithms by simulating annealing methods. The long-range electrostatic interactions were calculated by the particle mesh Ewald (PME) methods with a cutoff radius of $8.0 \AA[43,44]$. The simulation time step was set as $2.0 \mathrm{fs}$. The simulation trajectories were saved after $100 \mathrm{ps}$ and finally run for $20 \mathrm{~ns}$ by following the constant pressure and Berendsen thermostat [45]. The simulation trajectories were used to calculate the root-meansquare deviation, solvent accessible surface area, radius of gyration, and hydrogen bond [46-54].

\section{Results}

3.1. GC-MS Analysis. In this work, methanol was utilized as the solvent for extraction, resulting in a $1.34 \%$ yield. The GCMS analysis of the A. capitiformis stem methanolic extract revealed 49 compounds with different retention times and peak areas (Table 1 and Figure S1). The methanolic extract contained the following identified compounds: stigmast-4en-3-one (20.78\%, RT: 58.161); hexadeca-2,6,10,14-tetraen1-ol, 3,7,11,16-tetramethyl-, (E,E,E)- (18.36\%, RT: 51.477); ursa-9(11),12-dien-3-one (10.35\%, RT: 58.55); ursa9(11),12-dien-3-ol (6.89\%, RT: 55.077); 2-(2-hydroxy-2phenylethyl)-3,5,6-trimethylpyrazine (5.21\%, RT: 22.438); longipinane, (E)- (3.86\%, RT: 61.245); urs-12-ene $(2.95 \%$, RT: 57.274); 2-hydrazino-8-hydroxy-4-phenylquinoline (2.29\%, RT: 50.688); and friedelin (2.01\%, RT: 59.511).

3.2. Network Construction and Biological Process Analysis. A KEGG pathway analysis of potential target genes (IL1R1, IRAK4, MYD88, TIRAP, TLR4, and TRAF6) revealed signaling pathways related with anti-inflammatory effects (see Table 2). Figure S2 shows the PPI network with 12 proteins (IL1R1, IRAK2, MYD88, IRAK4, IRAK2, MYD88, TIRAP, TRAF6, TLR3, TLR4, TLR5, and TLR6), all of which have anti-inflammatory effects. Tables $\mathrm{S} 1$ and $\mathrm{S} 2$ represent the biological processes and molecular functions of the genes interacting with the compounds, respectively. Proteinprotein interaction (PPI) status of 10 proteins with coexpression is demonstrated in Table S3.

3.3. Molecular Docking and Simulation. A total of 47 compounds docking results are presented in Tables 3-8, which show the findings. Aspirin has been used as a positive control for this study. This study's findings reveal that binding energies of most of the ligands to receptors are negative, as later validated by MM-GBSA analysis. The proteins and ligands' molecular interaction is presented in Supplementary Materials (Tables S4-S9). To better understand the docking score, we have studied the ligand efficiency, which demonstrated excellent support for molecular docking scores. The molecular dynamics simulation of the targeted receptors (IL1R1, IRAK4, MYD88, TIRAP, TLR4, and TRAF6) against the best stable compounds is presented in Figures 1-6. 
TABLe 1: GC-MS analysis of the methanolic extract of Argyreia capitiformis stem.

\begin{tabular}{|c|c|c|c|c|c|}
\hline $\begin{array}{l}\text { Sl. } \\
\text { no. }\end{array}$ & $\mathrm{RT}(\min )$ & Area & PA (\%) & Compounds & $\begin{array}{l}\text { MW } \\
\text { (amu) }\end{array}$ \\
\hline 1 & 7.081 & 579298 & 0.105693 & Methylcyclohexane & 98.11 \\
\hline 2 & 9.965 & 3399237 & 0.620188 & Phenol & 94.042 \\
\hline 3 & 10.079 & 1001603 & 0.182742 & Sulcatone & 126.104 \\
\hline 4 & 10.148 & 780660 & 0.142431 & Butanoic acid, 2,3-dimethyl-, ethyl ester & 144.115 \\
\hline 5 & 10.348 & 865180 & 0.157852 & 1,2-Cyclohexanedione & 112.052 \\
\hline 6 & 11.601 & 1689097 & 0.308175 & 4-Methyl-1,5-heptadiene & 110.11 \\
\hline 7 & 16.104 & 925883 & 0.168927 & Catechol & 110.037 \\
\hline 8 & 16.373 & 5726885 & 1.044866 & Coumaran & 120.058 \\
\hline 9 & 18.359 & 4206254 & 0.767428 & Hydroquinone & 110.037 \\
\hline 10 & 18.879 & 2153554 & 0.392914 & p-Vinylguaiacol & 150.068 \\
\hline 11 & 19.412 & 630941 & 0.115115 & Gamma-pyronene & 136.125 \\
\hline 12 & 20.916 & 2138840 & 0.39023 & 4-Ethylresorcinol & 138.068 \\
\hline 13 & 22.438 & 28577337 & 5.213916 & 2-(2-Hydroxy-2-phenylethyl)-3,5,6-trimethylpyrazine & 242.142 \\
\hline 14 & 25.225 & 2487140 & 0.453777 & Ethanone, 1-(3,4-dimethoxyphenyl)- & 180.079 \\
\hline 15 & 25.563 & 4005631 & 0.730825 & Spathulenol & 220.183 \\
\hline 16 & 25.694 & 2465579 & 0.449843 & Caryophyllene oxide & 220.183 \\
\hline 17 & 26.295 & 639699 & 0.116713 & Cyclopentanecarboxaldehyde, 2-methyl-3-methylene- & 124.089 \\
\hline 18 & 27.314 & 1610820 & 0.293893 & Epiglobulol & 222.198 \\
\hline 19 & 27.668 & 1426632 & 0.260288 & 4(1H)-Pyrimidinone, 6-hydroxy- & 112.027 \\
\hline 20 & 28.126 & 1109370 & 0.202404 & Cyclododecanone, 2-methylene- & 194.167 \\
\hline 21 & 28.481 & 1911669 & 0.348783 & 2,3-Dehydro-4-oxo-7,8-dihydro-beta-ionone & 206.131 \\
\hline 22 & 28.767 & 1292504 & 0.235816 & Diepicedrene-1-oxide & 220.183 \\
\hline 23 & 29.213 & 10432471 & 1.903397 & Coniferol & 180.079 \\
\hline 24 & 33.848 & 7982629 & 1.456425 & n-Hexadecanoic acid & 256.24 \\
\hline 25 & 36.206 & 6006919 & 1.095958 & Methyl 6,9,12-hexadecatrienoate & 264.209 \\
\hline 26 & 36.44 & 14533536 & 2.651634 & Phytol & 296.308 \\
\hline 27 & 45.59 & 1109241 & 0.20238 & $\begin{array}{l}\text { 1,3,6,10-Cyclotetradecatetraene, 3,7,11-trimethyl-14-(1-methylethyl)-, [S- } \\
\text { (E,Z,E,E)]- }\end{array}$ & 272.25 \\
\hline 28 & 46.574 & 5222376 & 0.952819 & $(\mathrm{Z}, \mathrm{E})$-Farnesol & 222.198 \\
\hline 29 & 47.054 & 5942094 & 1.084131 & Geranyl acetate & 332.272 \\
\hline 30 & 47.215 & 4894168 & 0.892938 & Farnesol acetate & 264.209 \\
\hline 31 & 47.426 & 5740162 & 1.047289 & 3-Furaldehyde & 96.021 \\
\hline 32 & 47.781 & 1179960 & 0.215283 & trans-13-Docosenamide & 337.334 \\
\hline 33 & 48.542 & 6365219 & 1.16133 & Squalene & 410.391 \\
\hline 34 & 48.782 & 5112411 & 0.932756 & $\begin{array}{c}2^{\prime} \mathrm{H} \text {-Androsta-2,4,6-trieno [3,2-c]pyrazol-17.beta.-ol, 17-methyl-, acetate } \\
\text { (ester) }\end{array}$ & 366.231 \\
\hline 35 & 49.686 & 6003161 & 1.095273 & $\begin{array}{c}\text { Spiro[2H-indole- } 2,8^{\prime}\left(7^{\prime} \mathrm{H}\right) \text { - }[3,7] \text { methano }[2 \mathrm{H}] \text { furo }[4,3,2 \text {-hi]indolizine }] \text { - } \\
2^{\prime} \mathrm{a}\left(3^{\prime} \mathrm{H}\right) \text {-carboxylic acid, } 4^{\prime} \text {-ethylidene- } 1,3,4^{\prime}, 5^{\prime}, 8^{\prime}, 8^{\prime} \text { b-hexahydro-3-oxo- } \\
\text {, methyl ester }\end{array}$ & 366.158 \\
\hline 36 & 49.83 & 2892843 & 0.527797 & Chola-5,22-dien-3-ol, (3.beta.,22Z)- & 342.292 \\
\hline 37 & $\begin{array}{c}50.236 \text { and } \\
52.536\end{array}$ & $\begin{array}{c}2523311 \text { and } \\
4290679\end{array}$ & $\begin{array}{c}0.460376 \text { and } \\
0.782831\end{array}$ & Curan-17-oic acid, 2,16-didehydro-20-hydroxy-19-oxo-, methyl ester & 354.158 \\
\hline 38 & 50.47 & 7587558 & 1.384345 & Geranylgeraniol & 290.261 \\
\hline 39 & 50.688 & 12575157 & 2.294329 & 2-Hydrazino-8-hydroxy-4-phenylquinoline & 251.106 \\
\hline 40 & 51.477 & 100609821 & 18.35619 & Hexadeca-2,6,10,14-tetraen-1-ol, 3,7,11,16-tetramethyl-, (E,E,E)- & 290.261 \\
\hline 41 & 52.811 & 4449457 & 0.8118 & Cycloartenol & 426.386 \\
\hline 42 & 55.077 & 37780051 & 6.892945 & Ursa-9(11),12-dien-3-ol & 424.371 \\
\hline 43 & 57.274 & 16193192 & 2.954437 & Urs-12-ene & 410.391 \\
\hline 44 & 58.161 & 113880865 & 20.77749 & Stigmast-4-en-3-one & 412.371 \\
\hline 45 & 58.55 & 56736847 & 10.3516 & Ursa-9(11),12-dien-3-one & 422.355 \\
\hline 46 & 58.962 & 3509209 & 0.640253 & C(14a)-homo-27-nor-14-beta-gammaceran-3-alpha-ol & 428.402 \\
\hline 47 & 59.511 & 11017163 & 2.010074 & Friedelin & 426.386 \\
\hline 48 & 60.575 & 21170007 & 3.862453 & Longipinane, (E)- & 206.203 \\
\hline 49 & 61.245 & 2733077 & 0.498648 & Lanosterol & 426.386 \\
\hline
\end{tabular}

Note. MW: molecular weight; RT: retention time. 
TABLE 2: KEGG analysis of the genes targeted by compounds.

\begin{tabular}{|c|c|c|c|c|}
\hline $\begin{array}{l}\text { Pathway } \\
\text { ID }\end{array}$ & Pathway description & $\begin{array}{l}\text { Observed gene } \\
\text { count }\end{array}$ & $\begin{array}{c}\text { False } \\
\text { discovery rate }\end{array}$ & Matching proteins in network (labels) \\
\hline 05204 & Chemical carcinogenesis & 12 & $3.6 E-15$ & $\begin{array}{c}\text { SULT1A1, SULT1A2, SULT1A3, UGT1A1, UGT1A10, } \\
\text { UGT1A3, UGT1A4, UGT1A6, UGT1A7, UGT1A8, } \\
\text { UGT1A9, UGT2B15 }\end{array}$ \\
\hline 00053 & Ascorbate and aldarate metabolism & 9 & $1.21 E-14$ & $\begin{array}{c}\text { UGT1A1, UGT1A10, UGT1A3, UGT1A4, UGT1A6, } \\
\text { UGT1A7, UGT1A8, UGT1A9, UGT2B15 }\end{array}$ \\
\hline 00040 & $\begin{array}{l}\text { Pentose and glucuronate } \\
\text { interconversions }\end{array}$ & 9 & $1.49 E-13$ & $\begin{array}{c}\text { UGT1A1, UGT1A10, UGT1A3, UGT1A4, UGT1A6, } \\
\text { UGT1A7, UGT1A8, UGT1A9, UGT2B15 }\end{array}$ \\
\hline 00860 & $\begin{array}{l}\text { Porphyrin and chlorophyll } \\
\text { metabolism }\end{array}$ & 9 & $9.94 E-13$ & $\begin{array}{c}\text { UGT1A1, UGT1A10, UGT1A3, UGT1A4, UGT1A6, } \\
\text { UGT1A7, UGT1A8, UGT1A9, UGT2B15 }\end{array}$ \\
\hline 00983 & Drug metabolism-other enzymes & 9 & $1.27 E-12$ & $\begin{array}{c}\text { UGT1A1, UGT1A10, UGT1A3, UGT1A4, UGT1A6, } \\
\text { UGT1A7, UGT1A8, UGT1A9, UGT2B15 }\end{array}$ \\
\hline 00500 & Starch and sucrose metabolism & 9 & $4.62 E-12$ & $\begin{array}{c}\text { UGT1A1, UGT1A10, UGT1A3, UGT1A4, UGT1A6, } \\
\text { UGT1A7, UGT1A8, UGT1A9, UGT2B15 }\end{array}$ \\
\hline 00140 & Steroid hormone biosynthesis & 9 & $6.95 E-12$ & $\begin{array}{c}\text { UGT1A1, UGT1A10, UGT1A3, UGT1A4, UGT1A6, } \\
\text { UGT1A7, UGT1A8, UGT1A9, UGT2B15 }\end{array}$ \\
\hline 00830 & Retinol metabolism & 9 & $1.44 E-11$ & $\begin{array}{c}\text { UGT1A1, UGT1A10, UGT1A3, UGT1A4, UGT1A6, } \\
\text { UGT1A7, UGT1A8, UGT1A9, UGT2B15 }\end{array}$ \\
\hline 00982 & $\begin{array}{l}\text { Drug metabolism-cytochrome } \\
\text { P450 }\end{array}$ & 9 & $2.81 E-11$ & $\begin{array}{c}\text { UGT1A1, UGT1A10, UGT1A3, UGT1A4, UGT1A6, } \\
\text { UGT1A7, UGT1A8, UGT1A9, UGT2B15 }\end{array}$ \\
\hline 00980 & $\begin{array}{l}\text { Metabolism of xenobiotics by } \\
\text { cytochrome P450 }\end{array}$ & 9 & $4.52 E-11$ & $\begin{array}{c}\text { UGT1A1, UGT1A10, UGT1A3, UGT1A4, UGT1A6, } \\
\text { UGT1A7, UGT1A8, UGT1A9, UGT2B15 }\end{array}$ \\
\hline 04620 & $\begin{array}{l}\text { Toll-like receptor signaling } \\
\text { pathway }\end{array}$ & 9 & $1.83 E-09$ & $\begin{array}{c}\text { CD289, IRAK4, MYD88, TIRAP, TLR3, TLR4, TLR5, } \\
\text { TLR6, TRAF6 }\end{array}$ \\
\hline 05152 & Tuberculosis & 8 & $3.14 E-06$ & $\begin{array}{c}\text { CD289, IRAK2, IRAK4, MYD88, TIRAP, TLR4, TLR6, } \\
\text { TRAF6 }\end{array}$ \\
\hline 04064 & NF-kappa B signaling pathway & 6 & $1.35 E-05$ & IL1R1, IRAK4, MYD88, TIRAP, TLR4, TRAF6 \\
\hline 05142 & $\begin{array}{l}\text { Chagas disease (American } \\
\text { trypanosomiasis) }\end{array}$ & 6 & $2.09 E-05$ & CD289, IRAK4, MYD88, TLR4, TLR6, TRAF6 \\
\hline 05133 & Pertussis & 5 & $7.9 E-05$ & IRAK4, MYD88, TIRAP, TLR4, TRAF6 \\
\hline 05162 & Measles & 5 & 0.00154 & CD289, IRAK4, MYD88, TLR4, TRAF6 \\
\hline 05140 & Leishmaniasis & 4 & 0.00164 & IRAK4, MYD88, TLR4, TRAF6 \\
\hline 04210 & Apoptosis & 4 & 0.00332 & IL1R1, IRAK2, IRAK4, MYD88 \\
\hline 05144 & Malaria & 3 & 0.00825 & CD289, MYD88, TLR4 \\
\hline 05145 & Toxoplasmosis & 4 & 0.00895 & IRAK4, MYD88, TLR4, TRAF6 \\
\hline 05134 & Legionellosis & 3 & 0.0113 & MYD88, TLR4, TLR5 \\
\hline 05161 & Hepatitis B & 4 & 0.0182 & MYD88, TIRAP, TLR3, TLR4 \\
\hline 05164 & Influenza A & 4 & 0.0332 & IRAK4, MYD88, TLR3, TLR4 \\
\hline 05132 & Salmonella infection & 3 & 0.0337 & MYD88, TLR4, TLR5 \\
\hline 05168 & Herpes simplex infection & 4 & 0.0362 & CD289, MYD88, TLR3, TRAF6 \\
\hline
\end{tabular}

Bold indicates the main pathway and proteins responsible for this study.

\section{Discussion}

Plants have been used throughout the history of traditional medicine to induce a variety of biological effects, and extensive pharmaceutical resources have recently been devoted to the identification and investigation of new remedies, including those derived from plants. A critical issue encountered by researchers who perform phytoscience is that a single plant can harbor a wide range of bioactive chemicals $[55,56]$. The pharmaceutical industry relies on phytochemicals to develop new drugs and therapeutic agents. Finding natural bioactive components is the first step in developing novel drugs. Screening plant extracts for therapeutically active chemicals is a novel strategy. It is important to know that plants have a lot of different types of phytochemicals, which have a lot of different biological properties. These include antioxidant, anti-inflammatory, antidiarrhea, antiulcer, and anticancer activities [57-59]. Determining which compounds are responsible for the biological activities associated with plant materials can help understand toxicities, determine suitable doses, and identify ideal methods for compound extraction. The successful acquisition of components from plant materials depends primarily on the solvent used during the extraction process $[60,61]$. The stem methanolic extract was analyzed by GCMS analysis, and the results indicated 49 different chemicals with varying retention times and peak areas.

The network pharmacology analysis was performed to evaluate potential interactions between the identified chemical compounds and proteins, followed by multiple comparisons to determine the number of genes responsible for the anti-inflammatory effects, which was represented by the STITCH platform (Figure 7). Table 2 shows the results of KEGG pathway analysis performed on potential target 
TABLE 3: Docking scores and ligand efficiencies of compounds from the methanolic extract of Argyreia capitiformis stems binding with IL1R1 (PDB: 1ITB).

\begin{tabular}{|c|c|c|c|c|}
\hline \multirow{2}{*}{ Compounds } & \multicolumn{4}{|c|}{ IL1R1 (1ITB) } \\
\hline & DS* & MM-GBSA* & NHA & LE* \\
\hline Methylcyclohexane & -3.416 & -8.53477 & 7 & 1.22 \\
\hline Phenol & -4.567 & -20.6083 & 7 & 2.94 \\
\hline Sulcatone & -3.319 & -22.5701 & 9 & 2.51 \\
\hline Butanoic acid, 2,3-dimethyl-, ethyl ester & -3.695 & -22.6538 & 10 & 2.27 \\
\hline 1,2-Cyclohexanedione & -3.772 & -15.6443 & 8 & 1.96 \\
\hline 4-Methyl-1,5-heptadiene & -0.264 & -15.0204 & 8 & 1.88 \\
\hline Catechol & -4.755 & -24.1451 & 8 & 3.02 \\
\hline Coumaran & -4.368 & -18.8456 & 9 & 2.09 \\
\hline Hydroquinone & -4.703 & -21.6152 & 8 & 2.70 \\
\hline p-Vinylguaiacol & -3.965 & -17.0656 & 11 & 1.55 \\
\hline Gamma-pyronene & - & - & - & - \\
\hline 4-Ethylresorcinol & -4.791 & -19.4157 & 10 & 1.94 \\
\hline 2-(2-Hydroxy-2-phenylethyl)-3,5,6-trimethylpyrazine & -3.768 & -18.6465 & 18 & 1.04 \\
\hline Ethanone, 1-(3,4-dimethoxyphenyl)- & -5.147 & -24.0973 & 13 & 1.85 \\
\hline Spathulenol & - & - & - & - \\
\hline Caryophyllene oxide & - & - & - & - \\
\hline Cyclopentanecarboxaldehyde, 2-methyl-3-methylene- & -3.819 & -14.8427 & 9 & 1.65 \\
\hline Epiglobulol & - & - & - & - \\
\hline 4(1H)-Pyrimidinone, 6-hydroxy- & -5.289 & -22.2826 & 8 & 2.79 \\
\hline Cyclododecanone, 2-methylene- & -3.031 & -15.4537 & 14 & 1.10 \\
\hline 2,3-Dehydro-4-oxo-7,8-dihydro-beta-ionone & - & - & - & - \\
\hline Diepicedrene-1-oxide & -3.253 & -20.532 & 16 & 1.28 \\
\hline Coniferol & -4.551 & -25.8365 & 13 & 1.99 \\
\hline Methyl 6,9,12-hexadecatrienoate & -0.024 & -27.9609 & 19 & 1.47 \\
\hline Phytol & -0.765 & -31.806 & 21 & 1.51 \\
\hline 1,3,6,10-Cyclotetradecatetraene, 3,7,11-trimethyl-14-(1-methylethyl)-, [S-(E,Z,E,E)]- & - & - & - & - \\
\hline$(\mathrm{Z}, \mathrm{E})$-Farnesol & -1.134 & -28.241 & 16 & 1.77 \\
\hline Geranyl acetate & -3.607 & -33.7371 & 24 & 1.41 \\
\hline Farnesol, acetate & -1.636 & -37.7206 & 24 & 1.57 \\
\hline 3-Furaldehyde & -4.663 & -17.5816 & 7 & 2.51 \\
\hline trans-13-Docosenamide & -3.73 & -41.2676 & 24 & 1.72 \\
\hline Squalene & -3.241 & -26.5894 & 30 & 0.89 \\
\hline Chola-5,22-dien-3-ol, (3.beta.,22Z)- & -3.62 & -23.7437 & 25 & 0.95 \\
\hline Curan-17-oic acid, 2,16-didehydro-20-hydroxy-19-oxo-, methyl ester & - & - & - & - \\
\hline Geranylgeraniol & -1.699 & -26.6476 & 21 & 1.27 \\
\hline 2-Hydrazino-8-hydroxy-4-phenylquinoline & -5.348 & -26.1207 & 19 & 1.37 \\
\hline Cycloartenol & -2.877 & -17.9477 & 31 & 0.58 \\
\hline Ursa-9(11),12-dien-3-ol & - & - & - & - \\
\hline Urs-12-ene & -2.712 & -25.2104 & 30 & 0.84 \\
\hline Stigmast-4-en-3-one & -2.78 & -23.2611 & 30 & 0.78 \\
\hline Ursa-9(11),12-dien-3-one & - & - & - & - \\
\hline C(14a)-Homo-27-nor-14-beta-gammaceran-3-alpha-ol & - & - & - & - \\
\hline Friedelin & - & - & - & - \\
\hline Longipinane, (E)- & - & - & - & - \\
\hline Lanosterol & -3.249 & -26.5783 & 31 & 0.86 \\
\hline Aspirin & -4.26 & -21.1433 & 13 & 1.63 \\
\hline
\end{tabular}

Note. DS: docking score; NHA: number of heavy atoms; LE: ligand efficiency. ${ }^{*}$ Results presented in kcal/mol.

genes, which identified signaling pathways associated with anti-inflammatory actions. Analyses of biological processes and molecular functions, which identified proteins and pathways with significant values, were also performed (Table S1). A comparison of the compound-gene interaction network and the protein-protein interaction network was performed to reveal biological and molecular functions (Table S2). From a molecular and functional perspective, these findings can assist in understanding the computational rules of compounds that have the potential to treat diseases. For example, protein kinase C (PKC) binding is known to treat inflammatory diseases [62], and the present results showed that binding with TIRAP, UGT1A10, and UGT1A7 was significant $(p=0.0447)$ and similar to that for PKC. Also, retinoic acid binding has a great impact on the immune system and the inflammatory response. Our findings identified the genes UGT1A1, UGT1A3, UGT1A7, UGT1A8, and UGT1A9 as being the most significant interactions for the compounds in our extract $(p=2.69 E-06)$. 
TABLE 4: Docking scores and ligand efficiencies of compounds from the methanolic extract of Argyreia capitiformis stems binding with IRAK4 (PDB: 6EGA).

\begin{tabular}{|c|c|c|c|c|}
\hline \multirow{2}{*}{ Compounds } & \multicolumn{4}{|c|}{ IRAK4 (6EGA) } \\
\hline & DS* & MM-GBSA* & NHA & $\mathrm{LE}^{*}$ \\
\hline Methylcyclohexane & -5.343 & -28.5422 & 7 & 4.08 \\
\hline Phenol & -6.799 & -30.4637 & 7 & 4.35 \\
\hline Sulcatone & -4.611 & -34.0534 & 9 & 3.78 \\
\hline Butanoic acid, 2,3-dimethyl-, ethyl ester & -5.338 & -27.5643 & 10 & 2.76 \\
\hline 1,2-Cyclohexanedione & -5.827 & -26.2939 & 8 & 3.29 \\
\hline 4-Methyl-1,5-heptadiene & -2.392 & -31.5398 & 8 & 3.94 \\
\hline Catechol & -6.363 & -35.5712 & 8 & 4.45 \\
\hline Coumaran & -7.179 & -34.9848 & 9 & 3.89 \\
\hline Hydroquinone & -6.568 & -30.1474 & 8 & 3.77 \\
\hline p-Vinylguaiacol & -6.906 & -45.639 & 11 & 4.15 \\
\hline Gamma-pyronene & -5.242 & -25.8647 & 10 & 2.59 \\
\hline 4-Ethylresorcinol & -6.961 & -33.0487 & 10 & 3.30 \\
\hline 2-(2-Hydroxy-2-phenylethyl)-3,5,6-trimethylpyrazine & -7.589 & -47.6142 & 18 & 2.65 \\
\hline Ethanone, 1-(3,4-dimethoxyphenyl)- & -7.704 & -38.3452 & 13 & 2.95 \\
\hline Spathulenol & - & - & - & - \\
\hline Caryophyllene oxide & - & - & - & - \\
\hline Cyclopentanecarboxaldehyde, 2-methyl-3-methylene- & -6.889 & -34.1045 & 9 & 3.79 \\
\hline Epiglobulol & - & - & - & - \\
\hline 4(1H)-Pyrimidinone, 6-hydroxy- & -6.247 & -19.6834 & 8 & 2.46 \\
\hline Cyclododecanone, 2-methylene- & - & - & - & - \\
\hline 2,3-Dehydro-4-oxo-7,8-dihydro-beta-ionone & -6.224 & -26.8822 & 15 & 1.79 \\
\hline Diepicedrene-1-oxide & - & - & - & - \\
\hline Coniferol & -7.225 & -49.0664 & 13 & 3.77 \\
\hline Methyl 6,9,12-hexadecatrienoate & -3.841 & -51.8616 & 19 & 2.73 \\
\hline Phytol & -4.092 & -37.6416 & 21 & 1.79 \\
\hline 1,3,6,10-Cyclotetradecatetraene, 3,7,11-trimethyl-14-(1-methylethyl)-, [S-(E,Z,E,E)]- & - & - & - & - \\
\hline$(\mathrm{Z}, \mathrm{E})$-Farnesol & -4.144 & -53.635 & 16 & 3.35 \\
\hline Geranyl acetate & -7.613 & -46.7163 & 24 & 1.95 \\
\hline Farnesol acetate & -4.638 & -48.5373 & 24 & 2.02 \\
\hline 3-Furaldehyde & -6.301 & -28.4227 & 7 & 4.06 \\
\hline trans-13-Docosenamide & -6.62 & -55.939 & 24 & 2.33 \\
\hline Squalene & -7.603 & -73.1988 & 30 & 2.44 \\
\hline Chola-5,22-dien-3-ol, (3.beta.,22Z)- & -7.25 & -36.8492 & 25 & 1.47 \\
\hline Curan-17-oic acid, 2,16-didehydro-20-hydroxy-19-oxo-, methyl ester & - & - & - & - \\
\hline Geranylgeraniol & -4.383 & -54.1471 & 21 & 2.58 \\
\hline 2-Hydrazino-8-hydroxy-4-phenylquinoline & -6.639 & -35.0611 & 19 & 1.85 \\
\hline Cycloartenol & -3.914 & -28.0451 & 31 & 0.90 \\
\hline Ursa-9(11),12-dien-3-ol & - & - & - & - \\
\hline Urs-12-ene & -5.048 & -24.8357 & 30 & 0.83 \\
\hline Stigmast-4-en-3-one & -7.199 & -25.6365 & 30 & 0.85 \\
\hline Ursa-9(11),12-dien-3-one & - & - & - & - \\
\hline C(14a)-homo-27-nor-14-beta-gammaceran-3-alpha-ol & - & - & - & - \\
\hline Friedelin & - & - & - & - \\
\hline Longipinane, (E)- & - & - & - & - \\
\hline Lanosterol & -6.524 & -29.9751 & 31 & 0.97 \\
\hline Aspirin & -6.798 & -31.0373 & 13 & 2.39 \\
\hline
\end{tabular}

Note. DS: docking score; NHA: number of heavy atoms; LE: ligand efficiency. ${ }^{*}$ Results presented in kcal/mol.

As shown in Table 2, KEGG pathway analysis for inflammatory responses identified IL1R1, IRAK4, MYD88, TIRAP, TLR4, and TRAF6 in the NF- $\kappa \mathrm{B}$ signaling pathway, which has a $p$-value of $1.35 E-05$. Similar results were demonstrated for the inflammatory response in the biological process analysis, which identified the MYD88-dependent TLR signaling pathway $(p=2.19 E-07 ;$ IRAK2, IRAK4, MYD88, TIRAP, TLR4, TLR5, TLR6, and TRAF6), the TLR4 signaling pathway $(p=9.14 E-07$; IRAK2, IRAK4, MYD88, TIRAP, TLR3, TLR4, TLR6, and TRAF6), the TLR1:
TLR2 signaling pathway $(p=2.31 E-06$; IRAK2, IRAK4, MYD88, TIRAP, TLR4, TLR6, and TRAF6), the TLR 2 pathway $(p=2.54 E-06$; IRAK2, IRAK4, MYD88, TIRAP, TLR4, TLR6, and TRAF6), the TLR signaling pathway $(p=3.15 E-06 ;$ IRAK2, IRAK4, MYD88, TIRAP, TLR3, TLR4, TLR5, and TRAF6), positive regulation of interleukin6 production $(p=2.06 E-05 ; M Y D 88, T I R A P, T L R 3, T L R 4$, TLR6, and TRAF6), NF- $\kappa \mathrm{B}$ signaling $(p=0.000393$; IRAK2, TIRAP, TLR3, TLR4, and TRAF6), NF- $\kappa \mathrm{B}$ signaling $(p=0.000601 ;$ IRAK4, MYD88, TIRAP, TLR3, TLR4, TLR6, 
TABLE 5: Docking scores and ligand efficiencies of compounds from the methanolic extract of Argyreia capitiformis stems binding with MYD88 (PDB: 4EO7).

\begin{tabular}{|c|c|c|c|c|}
\hline \multirow{2}{*}{ Compounds } & \multicolumn{4}{|c|}{ MYD88 (4EO7) } \\
\hline & DS* & MM-GBSA* & NHA & $\mathrm{LE}^{*}$ \\
\hline Methylcyclohexane & -2.691 & -2.45925 & 7 & 0.35 \\
\hline Phenol & -3.654 & -5.09725 & 7 & 0.73 \\
\hline Sulcatone & -1.726 & -8.41174 & 9 & 0.93 \\
\hline Butanoic acid, 2,3-dimethyl-, ethyl ester & -1.597 & -2.95307 & 10 & 0.29 \\
\hline 1,2-Cyclohexanedione & -3.341 & -6.76768 & 8 & 0.85 \\
\hline 4-Methyl-1,5-heptadiene & 0.155 & -14.7508 & 8 & 1.84 \\
\hline Catechol & -3.771 & -8.25542 & 8 & 1.03 \\
\hline Coumaran & - & - & - & - \\
\hline Hydroquinone & -3.855 & -12.4109 & 8 & 1.55 \\
\hline p-Vinylguaiacol & -3.207 & -14.8801 & 11 & 1.35 \\
\hline Gamma-pyronene & - & - & - & - \\
\hline 4-Ethylresorcinol & -4.822 & -13.2875 & 10 & 1.33 \\
\hline 2-(2-Hydroxy-2-phenylethyl)-3,5,6-trimethylpyrazine & -3.247 & -32.6507 & 18 & 1.81 \\
\hline Ethanone, 1-(3,4-dimethoxyphenyl)- & -3.317 & -10.222 & 13 & 0.79 \\
\hline Spathulenol & - & - & - & - \\
\hline Caryophyllene oxide & -2.647 & -17.446 & 16 & 1.09 \\
\hline Cyclopentanecarboxaldehyde, 2-methyl-3-methylene- & - & - & - & - \\
\hline Epiglobulol & - & - & - & - \\
\hline $4(1 \mathrm{H})$-pyrimidinone, 6-hydroxy- & -4.189 & -6.98163 & 8 & 0.87 \\
\hline Cyclododecanone, 2-methylene- & - & - & - & - \\
\hline 2,3-Dehydro-4-oxo-7,8-dihydro-beta-ionone & - & - & - & - \\
\hline Diepicedrene-1-oxide & - & - & - & - \\
\hline Coniferol & -3.03 & -22.5252 & 13 & 1.73 \\
\hline Methyl 6,9,12-hexadecatrienoate & - & - & - & - \\
\hline Phytol & 0.454 & -26.4647 & 21 & 1.26 \\
\hline 1,3,6,10-Cyclotetradecatetraene, 3,7,11-trimethyl-14-(1-methylethyl)-, [S-(E,Z,E,E)]- & - & - & - & - \\
\hline$(\mathrm{Z}, \mathrm{E})$-Farnesol & 0.368 & -26.3771 & 16 & 1.65 \\
\hline Geranyl acetate & -2.457 & -29.1004 & 24 & 1.21 \\
\hline Farnesol, acetate & 0.023 & -20.0217 & 24 & 0.83 \\
\hline 3-Furaldehyde & - & - & - & - \\
\hline trans-13-Docosenamide & -2.916 & -42.0383 & 24 & 1.75 \\
\hline Squalene & -2.59 & -40.4505 & 30 & 1.35 \\
\hline Chola-5,22-dien-3-ol, (3.beta.,22Z)- & -2.236 & -7.69418 & 25 & 0.31 \\
\hline Curan-17-oic acid, 2,16-didehydro-20-hydroxy-19-oxo-, methyl ester & - & - & - & - \\
\hline Geranylgeraniol & 0.094 & -25.417 & 21 & 1.21 \\
\hline 2-Hydrazino-8-hydroxy-4-phenylquinoline & -3.068 & -7.45669 & 19 & 0.39 \\
\hline Cycloartenol & -1.726 & -21.1763 & 31 & 0.68 \\
\hline Ursa-9(11),12-dien-3-ol & - & - & - & - \\
\hline Urs-12-ene & -1.704 & -11.5462 & 30 & 0.38 \\
\hline Stigmast-4-en-3-one & -1.772 & -19.7209 & 30 & 0.66 \\
\hline Ursa-9(11),12-dien-3-one & - & - & - & - \\
\hline $\mathrm{C}(14 \mathrm{a})$-homo-27-nor-14-beta-gammaceran-3-alpha-ol & - & - & - & - \\
\hline Friedelin & - & - & - & - \\
\hline Longipinane, (E)- & -2.592 & -11.7102 & 15 & 0.78 \\
\hline Lanosterol & -1.84 & -21.6645 & 31 & 0.69 \\
\hline Aspirin & -1.659 & -5.81427 & 13 & 0.45 \\
\hline
\end{tabular}

Note. DS: docking score; NHA: number of heavy atoms; LE: ligand efficiency. ${ }^{*}$ Results presented in (kcal/mol).

and TRAF6), TLR5 signaling pathway ( $p=0.000712 ;$ IRAK2, IRAK4, MYD88, TLR5, and TRAF6), and TLR10 signaling pathway $(p=0.000712$; IRAK2, IRAK4, MYD88, TLR5, TRAF6). Six genes were identified as being associated with the NF- $\kappa \mathrm{B}$ signaling pathway, suggesting anti-inflammatory effects, in support of a previous study that identified the inhibition of NF- $\kappa$ B functional pathways in RAW 264.7 cells following treatment with a methanolic extract of A. capitiformis leaves [9].
Although a remarkable amount of functional and structural data has been compiled for each identified protein, our knowledge regarding protein-protein relationships remain scattered. The purpose of the STRING database is the collection, scoring, integration, and complementation with computational predictions for all public sources of PPIs [63-66]. In the present study, 10 proteins (IL1R1, IRAK4, IRAK2, MYD88, TIRAP, TRAF6, TLR3, TLR4, TLR5, and TLR6) were used to analyze a PPI network (Figure S2), which 
TABLE 6: Docking scores and ligand efficiencies of compounds from the methanolic extract of Argyreia capitiformis stems binding with TIRAP (PDB: 4FZ5).

\begin{tabular}{|c|c|c|c|c|}
\hline \multirow{2}{*}{ Compounds } & \multicolumn{4}{|c|}{ TIRAP (4FZ5) } \\
\hline & DS* & MM-GBSA* & NHA & $\mathrm{LE}^{*}$ \\
\hline Methylcyclohexane & -3.814 & -19.0091 & 7 & 2.72 \\
\hline Phenol & -4.448 & -21.4312 & 7 & 3.06 \\
\hline Sulcatone & -2.879 & -15.5041 & 9 & 1.72 \\
\hline Butanoic acid, 2,3-dimethyl-, ethyl ester & -3.225 & -19.8212 & 10 & 1.98 \\
\hline 1,2-Cyclohexanedione & -4.658 & -18.7199 & 8 & 2.34 \\
\hline 4-Methyl-1,5-heptadiene & -0.875 & -18.4864 & 8 & 2.31 \\
\hline Catechol & -4.501 & -22.731 & 8 & 2.84 \\
\hline Coumaran & -4.133 & -19.5103 & 9 & 2.17 \\
\hline Hydroquinone & -5.072 & -21.6796 & 8 & 2.71 \\
\hline p-Vinylguaiacol & -3.458 & -17.6974 & 11 & 1.61 \\
\hline Gamma-pyronene & -3.944 & -18.1058 & 10 & 1.81 \\
\hline 4-Ethylresorcinol & -5.971 & -29.5938 & 10 & 2.96 \\
\hline 2-(2-Hydroxy-2-phenylethyl)-3,5,6-trimethylpyrazine & -4.304 & -25.9223 & 18 & 1.44 \\
\hline Ethanone, 1-(3,4-dimethoxyphenyl)- & -5.243 & -24.616 & 13 & 1.89 \\
\hline Spathulenol & - & - & - & - \\
\hline Caryophyllene oxide & - & - & - & - \\
\hline Cyclopentanecarboxaldehyde, 2-methyl-3-methylene- & -4.868 & -20.8151 & 9 & 2.31 \\
\hline Epiglobulol & - & - & - & - \\
\hline $4(1 \mathrm{H})$-pyrimidinone, 6-hydroxy- & -4.845 & -15.1979 & 8 & 1.89 \\
\hline Cyclododecanone, 2-methylene- & -2.974 & -20.1319 & 14 & 1.44 \\
\hline 2,3-Dehydro-4-oxo-7,8-dihydro-beta-ionone & -4.032 & -21.7031 & 15 & 1.45 \\
\hline Diepicedrene-1-oxide & - & - & - & - \\
\hline Coniferol & -4.855 & -32.2717 & 13 & 2.48 \\
\hline Methyl 6,9,12-hexadecatrienoate & 0.105 & -31.8802 & 19 & 1.68 \\
\hline Phytol & 0.856 & -29.859 & 21 & 1.42 \\
\hline 1,3,6,10-Cyclotetradecatetraene, 3,7,11-trimethyl-14-(1-methylethyl)-, [S-(E,Z,E,E)]- & - & - & - & - \\
\hline$(\mathrm{Z}, \mathrm{E})$-Farnesol & 0.289 & -15.1735 & 16 & 0.95 \\
\hline Geranyl acetate & -1.574 & -25.783 & 24 & 1.07 \\
\hline Farnesol, acetate & 0.333 & -14.545 & 24 & 0.61 \\
\hline 3-Furaldehyde & -4.332 & -17.5272 & 7 & 2.50 \\
\hline trans-13-Docosenamide & -1.763 & -31.4792 & 24 & 1.31 \\
\hline Squalene & -1.678 & -23.5817 & 30 & 0.79 \\
\hline Chola-5,22-dien-3-ol, (3.beta.,22Z)- & -2.807 & -23.4983 & 25 & 0.94 \\
\hline Curan-17-oic acid, 2,16-didehydro-20-hydroxy-19-oxo-, methyl ester & - & - & - & - \\
\hline Geranylgeraniol & 0.085 & -9.16848 & 21 & 0.44 \\
\hline 2-Hydrazino-8-hydroxy-4-phenylquinoline & -4.084 & -24.5226 & 19 & 1.29 \\
\hline Cycloartenol & -1.963 & -29.9984 & 31 & 0.97 \\
\hline Ursa-9(11),12-dien-3-ol & - & - & - & - \\
\hline Urs-12-ene & -2.561 & -20.6408 & 30 & 0.69 \\
\hline Stigmast-4-en-3-one & - & - & - & - \\
\hline Ursa-9(11),12-dien-3-one & -3.348 & -39.9359 & 31 & 1.29 \\
\hline C(14a)-homo-27-nor-14-beta-gammaceran-3-alpha-ol & - & - & - & - \\
\hline Friedelin & - & - & - & - \\
\hline Longipinane, (E)- & - & - & - & - \\
\hline Lanosterol & -1.716 & -27.5587 & 31 & 0.89 \\
\hline Aspirin & -4.343 & -23.6086 & 13 & 1.82 \\
\hline
\end{tabular}

Note. DS: docking score; NHA: number of heavy atoms; LE: ligand efficiency. ${ }^{*}$ Results presented in kcal/mol.

were all significantly $(p<1.0 e-16)$ related to anti-inflammatory functions. Signals from the various TLRs are diverse, but all TLRs are activated by the binding of TIR-containing adaptor proteins (e.g., TIRAP activates MYD88, and TRAM activates TRIF). IRAK4, IRAK1, IRAK2, and TRAF6 are all activated by MYD88. MYD88 binding results in the phosphorylation and release of IRAK1 from the cell membrane into the cytoplasm, where it interacts with and activates the transforming growth factor- $\beta$-activated kinase 1 (TAK1) downstream. TAK1 activates the IKK $\beta$-to-I $\kappa \mathrm{B}-\alpha$-to-NF- $\kappa \mathrm{B}$ pathway, inducing the transcription of proinflammatory genes. TAK1 also influences gene expression by activating mitogen-activated protein kinase (MAPK) cascades. TRAF6, $\beta$ RIP1, and TAK1 are activated by the binding of TLR with TRIF, resulting in the activation of MAPK, interferon regulatory factor 3 (IRF3), NF- $\kappa \mathrm{B}$, and interferon- transcription activation. The TRIF pathway also promotes the release of proinflammatory cytokines, although to a lesser 
TABLE 7: Docking scores and ligand efficiencies of compounds from the methanolic extract of Argyreia capitiformis stems binding with TLR4 (PDB: 3FXI).

\begin{tabular}{|c|c|c|c|c|}
\hline \multirow{2}{*}{ Compounds } & \multicolumn{4}{|c|}{ TLR4 (3FXI) } \\
\hline & DS* & MM-GBSA* & NHA & $\mathrm{LE}^{*}$ \\
\hline Methylcyclohexane & -5.225 & -25.2088 & 7 & 3.60 \\
\hline Phenol & -6.387 & -32.1845 & 7 & 4.59 \\
\hline Sulcatone & -5.227 & -37.5653 & 9 & 4.17 \\
\hline Butanoic acid, 2,3-dimethyl-, ethyl ester & -4.588 & -32.241 & 10 & 3.22 \\
\hline 1,2-Cyclohexanedione & -6.231 & -26.8612 & 8 & 3.36 \\
\hline 4-Methyl-1,5-heptadiene & -2.573 & -35.6785 & 8 & 4.45 \\
\hline Catechol & -6.224 & -35.1395 & 8 & 4.39 \\
\hline Coumaran & -6.217 & -31.1228 & 9 & 3.46 \\
\hline Hydroquinone & -5.879 & -32.0765 & 8 & 4.01 \\
\hline p-Vinylguaiacol & -6.497 & -41.7567 & 11 & 3.79 \\
\hline Gamma-pyronene & -5.859 & -28.5764 & 10 & 2.86 \\
\hline 4-Ethylresorcinol & -7.636 & -30.7191 & 10 & 3.07 \\
\hline 2-(2-Hydroxy-2-phenylethyl)-3,5,6-trimethylpyrazine & -7.775 & -47.2615 & 18 & 2.63 \\
\hline Ethanone, 1-(3,4-dimethoxyphenyl)- & -7.127 & -35.528 & 13 & 2.73 \\
\hline Spathulenol & - & - & - & - \\
\hline Caryophyllene oxide & - & - & - & - \\
\hline Cyclopentanecarboxaldehyde, 2-methyl-3-methylene- & -6.018 & -29.885 & 9 & 3.32 \\
\hline Epiglobulol & - & - & - & - \\
\hline $4(1 \mathrm{H})$-pyrimidinone, 6-hydroxy- & -5.805 & -25.5615 & 8 & 3.19 \\
\hline Cyclododecanone, 2-methylene- & - & - & - & - \\
\hline 2,3-Dehydro-4-oxo-7,8-dihydro-.beta.-ionone & -6.232 & -10.5536 & 15 & 0.70 \\
\hline Diepicedrene-1-oxide & - & - & - & - \\
\hline Coniferol & -7.058 & -52.0494 & 13 & 4.003 \\
\hline Methyl 6,9,12-hexadecatrienoate & -2.949 & -48.4222 & 19 & 2.55 \\
\hline Phytol & -3.527 & -48.9579 & 21 & 2.33 \\
\hline 1,3,6,10-Cyclotetradecatetraene, 3,7,11-trimethyl-14-(1-methylethyl)-, [S-(E,Z,E,E)]- & - & - & - & - \\
\hline$(\mathrm{Z}, \mathrm{E})$-Farnesol & -3.612 & -49.9903 & 16 & 3.12 \\
\hline Geranyl acetate & -6.867 & -47.3758 & 24 & 1.97 \\
\hline Farnesol, acetate & -4.062 & -56.2792 & 24 & 2.34 \\
\hline 3-Furaldehyde & -5.191 & -27.4652 & 7 & 3.92 \\
\hline trans-13-Docosenamide & -5.443 & -52.2716 & 24 & 2.18 \\
\hline Squalene & -6.729 & -40.8575 & 30 & 1.36 \\
\hline Chola-5,22-dien-3-ol, (3.beta.,22Z)- & - & - & - & - \\
\hline Curan-17-oic acid, 2,16-didehydro-20-hydroxy-19-oxo-, methyl ester & - & - & - & - \\
\hline Geranylgeraniol & -3.692 & -42.9074 & 21 & 2.04 \\
\hline 2-Hydrazino-8-hydroxy-4-phenylquinoline & -5.647 & 0.186151 & 19 & -0.009 \\
\hline Cycloartenol & - & - & - & - \\
\hline Ursa-9(11),12-dien-3-ol & - & - & - & - \\
\hline Urs-12-ene & - & - & - & - \\
\hline Stigmast-4-en-3-one & - & - & - & - \\
\hline Ursa-9(11),12-dien-3-one & - & - & - & - \\
\hline C(14a)-homo-27-nor-14-beta-gammaceran-3-alpha-ol & - & - & - & - \\
\hline Friedelin & - & - & - & - \\
\hline Longipinane, (E)- & - & - & - & - \\
\hline Lanosterol & - & - & - & - \\
\hline Aspirin & -6.329 & -35.694 & 13 & 2.75 \\
\hline
\end{tabular}

Note. DS: docking score; NHA: number of heavy atoms; LE: ligand efficiency. ${ }^{*}$ Results presented in kcal/mol.

degree than the MYD88 pathway. TLRs are a class of specific receptors that are key players in mounting an effective innate immune response to infection [67-69].

Subsequently, gene co-occurrence can be used to identify gene families whose patterns exhibit similarity across genomes. Three types of analyses have been used to examine genomes (neighborhood, fusion, gene co-occurrence) based on the systemic comparison of all-against-all genomes to evaluate the impacts of historical genome restructurings, genetic gains and losses, and gene fusion [70, 71]. In the present study, $100 \%$ sequence conservation was observed for the ten proteins (IL1R1, IRAK4, IRAK2, MYD88, TIRAP, TRAF6, TLR3, TLR4, TLR5, and TLR6) selected for the PPI network analysis, as shown in Figure S3. Additionally, gene coexpression was also studied in the present study, as summarized in Table S3 and Figure S4. The coexpression pathway is predicated by performing gene-by-gene correlation testing across many gene expression databases. 
TABLE 8: Docking scores and ligand efficiencies of compounds from the methanolic extract of Argyreia capitiformis stems binding with TRAF6 (PDB: 3HCT).

\begin{tabular}{|c|c|c|c|c|}
\hline \multirow{2}{*}{ Compounds } & \multicolumn{4}{|c|}{ TRAF6 (3HCT) } \\
\hline & DS* $^{*}$ & MM-GBSA* & NHA & LE* $^{*}$ \\
\hline Methylcyclohexane & -4.23 & -15.5462 & 7 & 2.22 \\
\hline Phenol & -4.75 & -17.0982 & 7 & 2.44 \\
\hline Sulcatone & -3.224 & -22.1931 & 9 & 2.47 \\
\hline Butanoic acid, 2,3-dimethyl-, ethyl ester & -3.328 & -17.6969 & 10 & 1.77 \\
\hline 1,2-Cyclohexanedione & -5.406 & -17.7531 & 8 & 2.22 \\
\hline 4-Methyl-1,5-heptadiene & -1.225 & -18.4899 & 8 & 2.31 \\
\hline Catechol & -4.53 & -18.205 & 8 & 2.28 \\
\hline Coumaran & -3.768 & -20.8505 & 9 & 2.32 \\
\hline Hydroquinone & -4.795 & -18.3408 & 8 & 2.29 \\
\hline p-Vinylguaiacol & -4.303 & -17.778 & 11 & 1.62 \\
\hline Gamma-pyronene & -4.133 & -21.4044 & 10 & 2.14 \\
\hline 4-Ethylresorcinol & -5.48 & -19.6712 & 10 & 1.97 \\
\hline 2-(2-Hydroxy-2-phenylethyl)-3,5,6-trimethylpyrazine & -5.119 & -41.8887 & 18 & 2.33 \\
\hline Ethanone, 1-(3,4-dimethoxyphenyl)- & -5.708 & -29.3694 & 13 & 2.26 \\
\hline Spathulenol & - & - & - & - \\
\hline Caryophyllene oxide & - & - & - & - \\
\hline Cyclopentanecarboxaldehyde, 2-methyl-3-methylene- & -5.198 & -20.7855 & 9 & 2.31 \\
\hline Epiglobulol & - & - & - & - \\
\hline $4(1 \mathrm{H})$-pyrimidinone, 6-hydroxy- & -5.483 & -15.5853 & 8 & 1.95 \\
\hline Cyclododecanone, 2-methylene- & -4.619 & -3.58279 & 14 & 0.26 \\
\hline 2,3-Dehydro-4-oxo-7,8-dihydro-beta-ionone & -4.327 & -25.9777 & 15 & 1.73 \\
\hline Diepicedrene-1-oxide & - & - & - & - \\
\hline Coniferol & -3.698 & -31.781 & 13 & 2.44 \\
\hline Methyl 6,9,12-hexadecatrienoate & 0.144 & -27.9098 & 19 & 1.47 \\
\hline Phytol & 1.098 & -20.446 & 21 & 0.97 \\
\hline 1,3,6,10-Cyclotetradecatetraene, 3,7,11-trimethyl-14-(1-methylethyl)-, [S-(E,Z,E,E)]- & - & - & - & - \\
\hline (Z,E)-Farnesol & -0.033 & -18.0454 & 16 & 1.13 \\
\hline Geranyl acetate & -2.742 & -22.1469 & 24 & 0.92 \\
\hline Farnesol, acetate & -0.582 & -27.6353 & 24 & 1.15 \\
\hline 3-Furaldehyde & -4.911 & -16.8696 & 7 & 2.41 \\
\hline trans-13-Docosenamide & -2.779 & -43.4455 & 24 & 1.81 \\
\hline Squalene & -2.1 & -40.8237 & 30 & 1.36 \\
\hline Chola-5,22-dien-3-ol, (3.beta.,22Z)- & -2.904 & -21.7666 & 25 & 0.87 \\
\hline Curan-17-oic acid, 2,16-didehydro-20-hydroxy-19-oxo-, methyl ester & - & - & - & - \\
\hline Geranylgeraniol & -0.313 & -26.3103 & 21 & 1.253 \\
\hline 2-Hydrazino-8-hydroxy-4-phenylquinoline & -4.282 & -21.7512 & 19 & 1.14 \\
\hline Cycloartenol & -2.32 & -17.571 & 31 & 0.57 \\
\hline Ursa-9(11),12-dien-3-ol & - & - & - & - \\
\hline Urs-12-ene & -2.153 & -13.0659 & 30 & 0.44 \\
\hline Stigmast-4-en-3-one & -2.229 & -18.7231 & 30 & 0.62 \\
\hline Ursa-9(11),12-dien-3-one & - & - & - & - \\
\hline C(14a)-homo-27-nor-14-beta-gammaceran-3.alpha.-ol & - & - & - & - \\
\hline Friedelin & - & - & - & - \\
\hline Longipinane, (E)- & - & - & - & - \\
\hline Lanosterol & -2.204 & -16.5677 & 31 & 0.53 \\
\hline Aspirin & -4.81 & -26.889 & 13 & 2.07 \\
\hline
\end{tabular}

Note. DS: docking score; NHA: number of heavy atoms; LE: ligand efficiency. ${ }^{*}$ Results presented in kcal/mol.

STRING reconstructs and maps this enormous series of experiments, which is stored on the NCBI database along with transcript data $[63,72]$. The present findings suggested co-expression among the ten selected proteins (IL1R1, IRAK4, IRAK2, MYD88, TIRAP, TRAF6, TLR3, TLR4, TLR5, and TLR6) in Homo sapiens. In addition, coexpression (transferred) of three more genes was observed in Gallus gallus, Mus musculus, and Rattus norvegicus.

Structure-based drug discovery is gradually becoming a key technique for facilitating the rapid and cost-effective discovery and optimization of lead compounds. The use of a rational, structure-based drug design strategy is more efficient than conventional drug development techniques because this approach seeks to understand the molecular basis of diseases and incorporates information regarding the biological target's 3D structure during the drug design process [37]. A molecular docking study was incorporated into the present study to predict the complex structure formed by ligand-protein binding and analyze the ligand's conformational space within the protein-binding site. A score function 


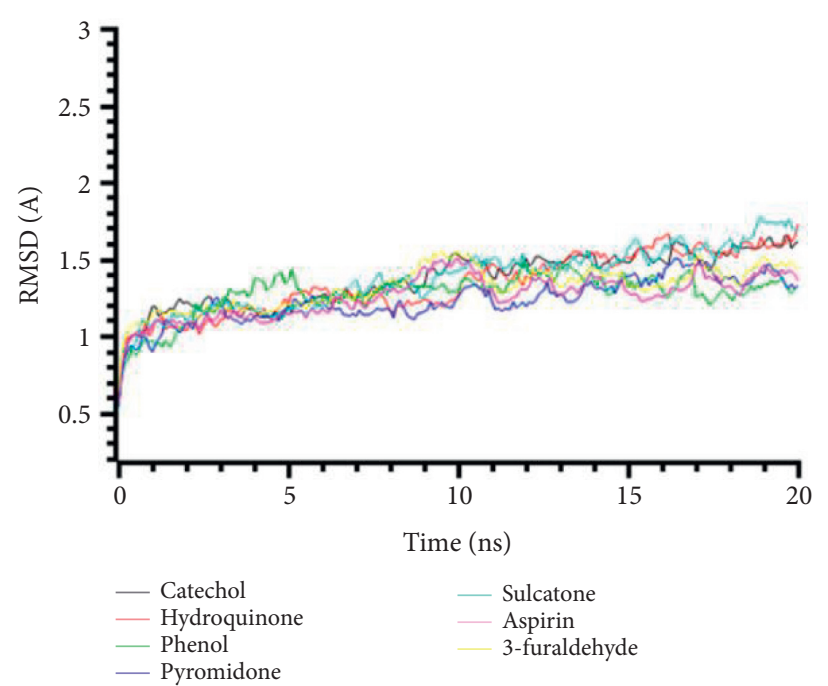

(a)

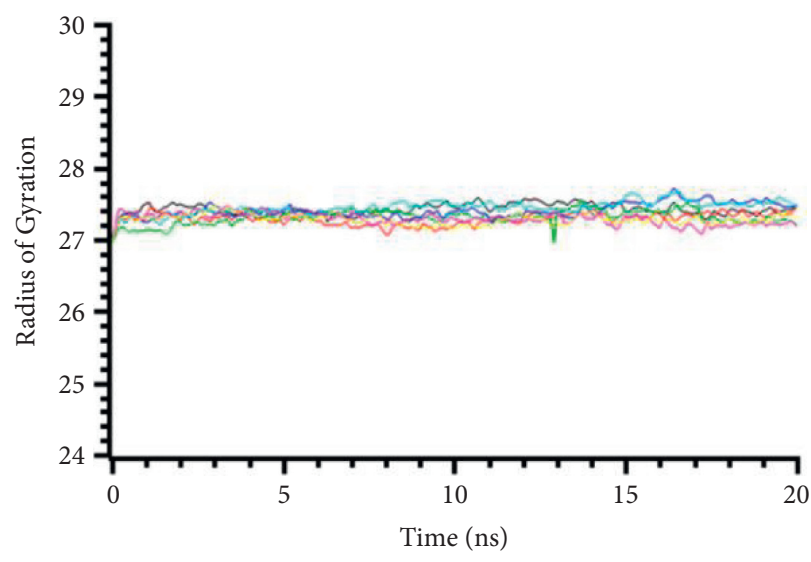

(c)

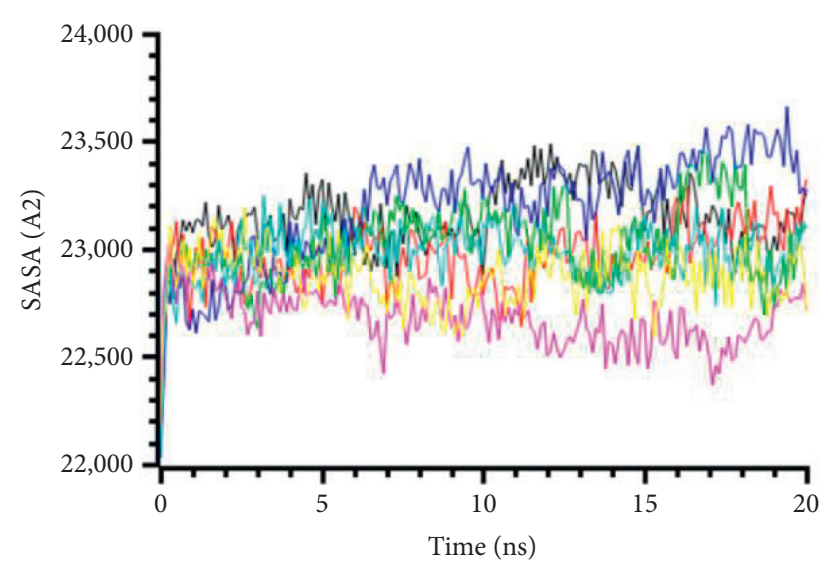

(b)

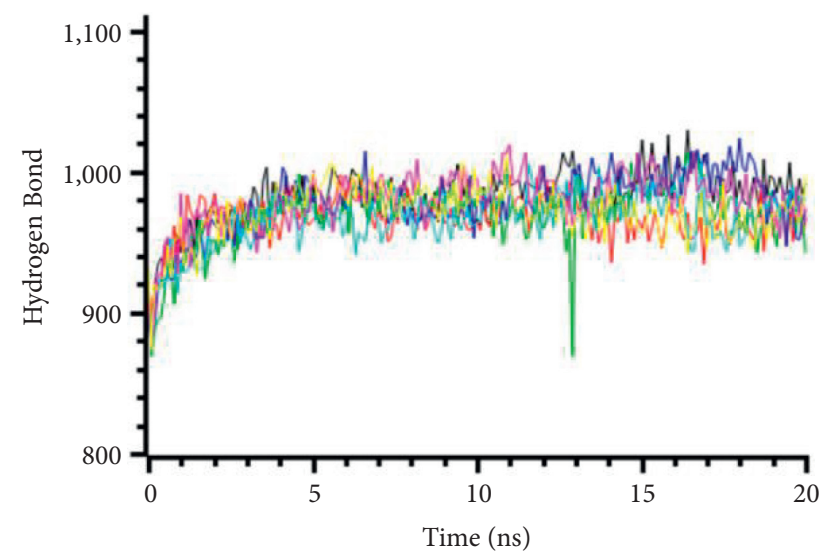

(d)

FIgURE 1: Molecular dynamics simulation of the best ligand efficiencies for compounds against IL1R1 (PDB: 1ITB). (a) Root-mean-square deviation of the complexes (RMSD). (b) Solvent accessible surface area (SASA). (c) Radius of gyration (Rg). (d) Hydrogen bond analysis from the simulation system.

is then used for each docking analysis to assess the free energy of the interaction between the protein and ligand $[37,73,74]$. Additionally, the LE is calculated, which can enrich docking functions and allow for the coordination between docking outcomes and experimental results. Critical information regarding a molecule's properties, such as the NHA, can then be combined into a single table [39].

IL-1 controls a range of innate immune pathways, making it a key regulator of inflammation [75]. Two IL-1 cell surface receptors and a decoy receptor have been identified, including IL1R1 and IL1R2. First, IL-1 binds with IL1R1, inducing the formation of a heterodimer between IL1R1 and either IL-1RAcP or IL1R3, followed by IL-1 receptor-associated kinase (IRAK) and MyD88. The inflammatory response induced by IL1R1 occurs when IL1R1 binds with either the IL- $1 \alpha$ or IL- $1 \beta$ ligands, whereas the T-lymphocytes, fibroblastic cells, epithelial cells, and endothelial cells have been indicated [76-78]. In the present study, selected six target proteins based on the network pharmacology analysis for the molecular docking study: IL1R1 (PDB:
1ITB), IRAK4 (PDB: 6EGA), and MYD88 (PDB: 4EO7) was used for the docking study. For IL1R1 (PDB: 1ITB), the majority of the 47 tested compounds demonstrated good docking scores, except for 12 compounds (Table 3). Among the 33 compounds with good docking scores, catechol (3.02), phenol (2.94), 4(1H)-pyrimidinone, 6-hydroxy- (2.79), hydroquinone (2.70), 3-furaldehyde (2.51), and sulcatone (2.51) showed the best ligand efficiencies, compared with the LE value of 1.63 for the positive control aspirin (Table S4 and Figure S5). The compounds with the best ligand efficiencies were found to interact with LYS-93 by H-bond (distances $>4 \AA$ ), and catechol interacted via two H-bonds. Similar findings were also observed for the positive control aspirin. According to an earlier study, the noncontiguous binding epitope containing LYS-93 was identified for IL-1 $\beta$ [79].

IRAK4 (PDB: 6EGA) also exhibited similar findings in the docking experiment, with only 12 compounds that did not display any interactions. Catechol (4.45), phenol (4.35), p-vinylguaiacol (4.15), methylcyclohexane (4.08), 3-furaldehyde (4.06), and 4-methyl-1,5-heptadiene (3.94) showed 


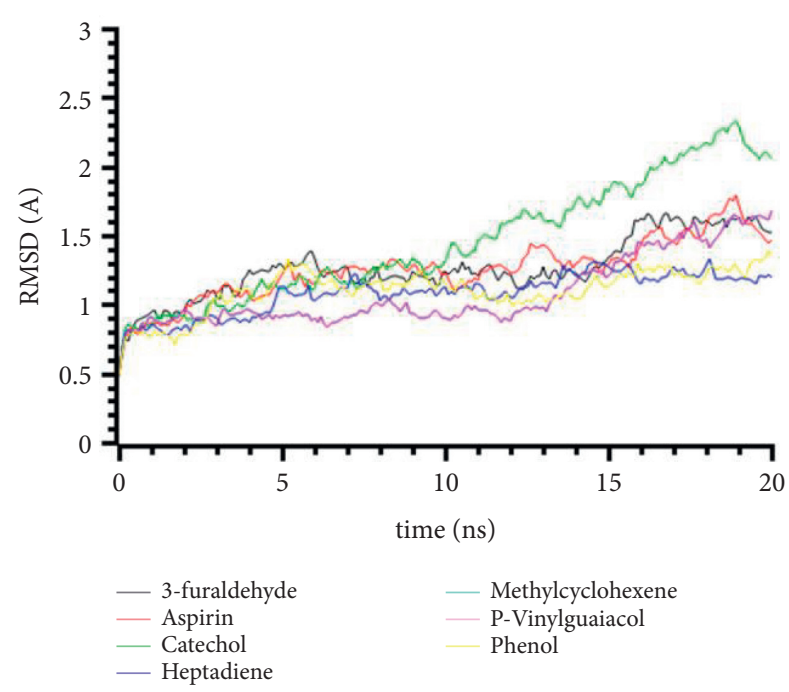

(a)

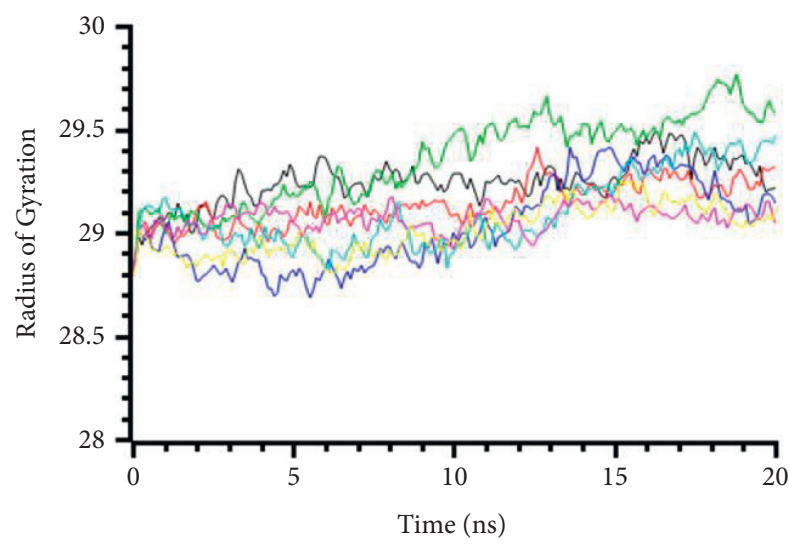

(c)

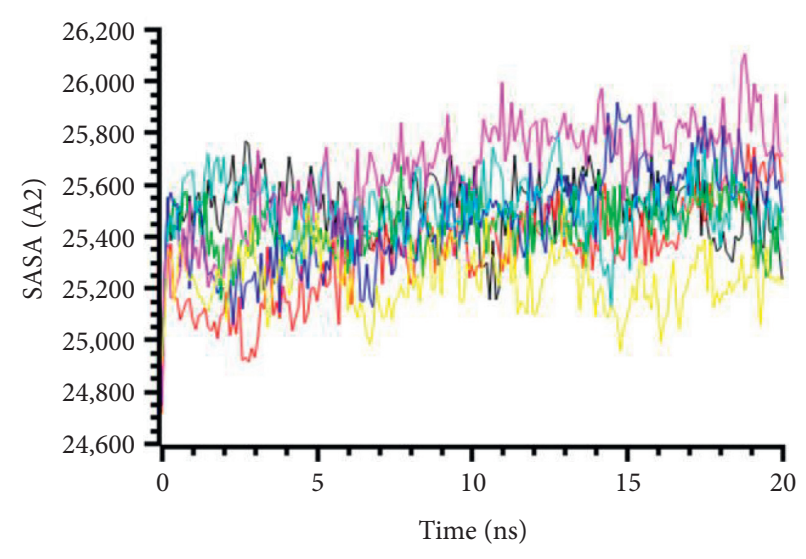

(b)

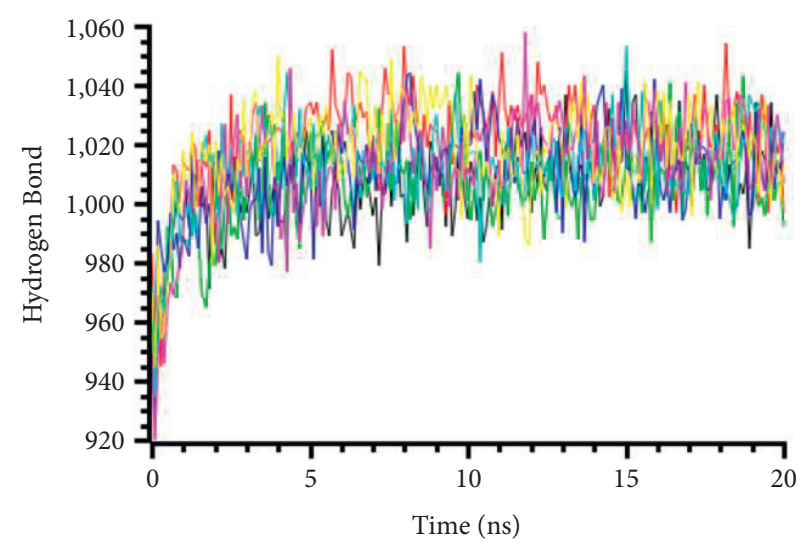

(d)

FIgURE 2: Two-dimensional representations of the best ligand efficiencies for compounds against IRAK4 (PDB: 6EGA). (a) Root-meansquare deviation of the complexes (RMSD). (b) Solvent accessible surface area (SASA). (c) Radius of gyration (Rg). (d) Hydrogen bond analysis from the simulation system.

the best LE values, as shown in Table 4. The LE value of aspirin was 2.39. The molecular interactions between the compounds with the best LE values and IRAK4 (PDB: 6EGA) are presented in Table S5 and Figure S6. ASP-329 formed H-bonds with catechol (4.02, 3.48 $)$, p-vinylguaiacol (4.05, $4.06 \AA)$, phenol $(3.49 \AA)$, and aspirin $(4.24 \AA)$, whereas no H-bond interactions were observed for 4-methyl-1,5-heptadiene or methylcyclohexane. Additionally, 3-furaldehyde interacted with MET-265 (4.0 ̊) via one H-bond. Remarkably, ASP329, MET-265, and LYS-213 were all reported to interact with IRAK4 in a previous study [32].

Additionally, MYD88 (PDB: 4EO7) was also studied as a potential target of the anti-inflammatory effects of the methanolic extract of $A$. capitiformis stems. In the molecular docking experiment, 29 compounds were found to interact with MYD88 (Table 5). The best LE values for MYD88 (PDB: 4EO7) were identified for 4-methyl-1,5-heptadiene (1.84), 2-(2-hydroxy-2-phenylethyl)-3,5,6-trimethylpyrazine (1.81), trans-13-docosenamide (1.75), coniferol (1.73), (Z, E)-farnesol (1.65), and hydroquinone (1.55). The positive control aspirin had an LE value of 0.45 , which was lower than the LE values for 24 of the identified compounds. The molecular interactions between the compounds with the best LE values and MYD88 (PDB: 4EO7) are presented in Table S6 and Figure S7. ASP-156 was reported to form H-bonds with coniferol, 2-(2-hydroxy-2-phenylethyl)3,5,6-trimethylpyrazine, and (Z,E)-farnesol, whereas hydroquinone interacted with ASP-156 through the formation of hydrophobic bonds (3.51 $\mathrm{\AA})$. No H-bond interactions were reported for 4-methyl-1,5-heptadiene. The other compound, trans-13-docosenamide, interacted with ARG160 (5.21 $\AA$ ) and GLU-159 (4.36 $\AA$ ) via H-bonds.

TIRAP, also known as MYD88 adapter-like (Mal), is an important link between MYD88 and the receptor complex formed by TLR2 and TLR4 activation following bacterial infection [32]. MYD88 activates IRAK1 and IRAK4 and eventually activates TRAF6, causing the prototypic inflammatory transcription factor NF- $\kappa \mathrm{B}$ to translocate to the nucleus [80, 81]. TIRAP is the second adaptor that has been identified to mediate NF- $\kappa \mathrm{B}$ activation through the activation 


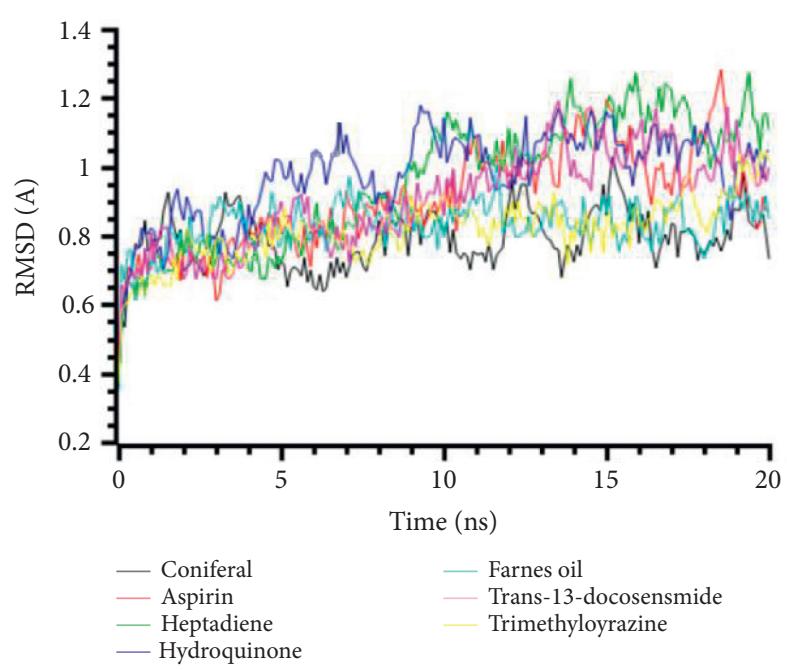

(a)

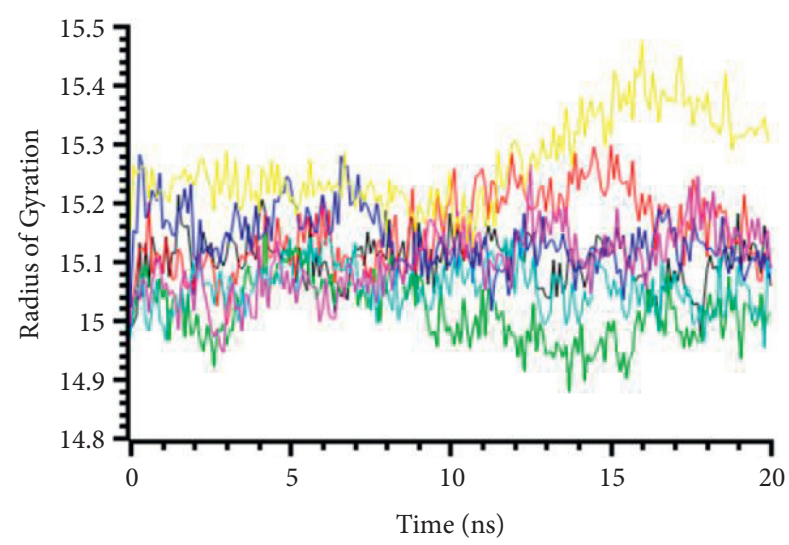

(c)

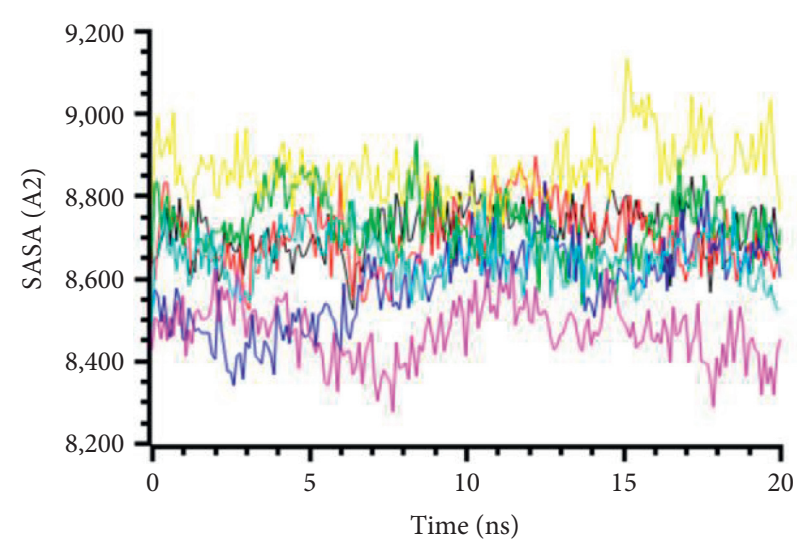

(b)

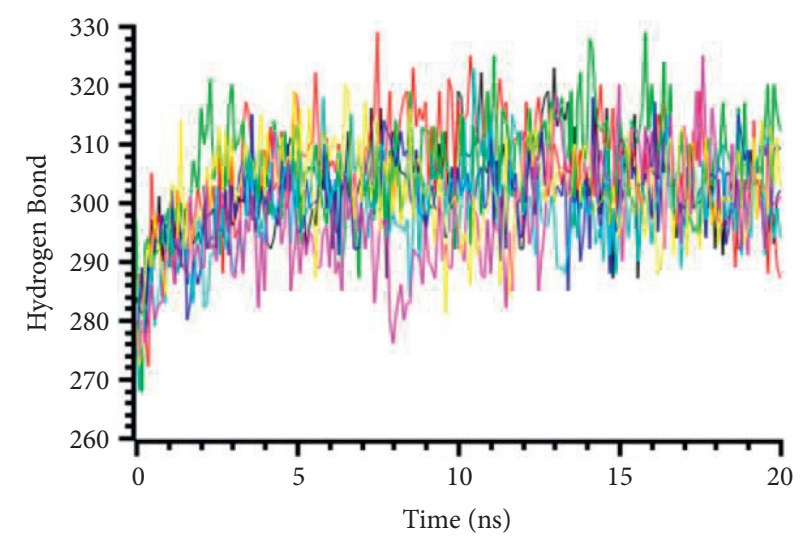

(d)

Figure 3: Two-dimensional representations of the best ligand efficiencies for compounds against MYD88 (4EO7). (a) Root-mean-square deviation of the complexes (RMSD). (b) Solvent accessible surface area (SASA). (c) Radius of gyration (Rg). (d) Hydrogen bond analysis from the simulation system.

of TLR4 and TLR2 signaling pathways [82-85]. The molecular docking of TIRAP (PDB: 4FZ5) was examined against the 45 compounds identified in the methanolic extract of A. capitiformis stems, of which 35 demonstrated interactions and 10 did not (Table 6). The best interacting compounds were phenol (3.06), 4-ethylresorcinol (2.96), catechol (2.84), methylcyclohexane (2.72), hydroquinone (2.71), and 3-furaldehyde (2.50), compared with the positive control aspirin, which exhibited an LE of 1.82 (Figure S8). The molecular interactions between the compounds with the best $L E$ values and TIRAP (PDB : 4FZ5) are presented in Table S7. LEU-107 formed H-bond with 3-furaldehyde, catechol, phenol, and aspirin, and a hydrophobic interaction was also observed for catechol, methylcyclohexane, and phenol. Another protein residue, LYS-210, interacted with 3-furaldehyde, 4-ethylresorcinol, hydroquinone, and phenol via hydrophobic interactions. In a previous study, LYS-210 was reported to form a hydrophobic interaction with NF- $\kappa \mathrm{B}$ [86].

In addition, TLR4 (PDB: 3FXI) was compared against 45 compounds found in the methanolic extract of A. capitiformis stems, although 17 compounds did not interact with TLR4 (Table 7). Phenol (4.59), 4-methyl-1,5heptadiene (4.45), catechol (4.39), sulcatone (4.17), hydroquinone (4.01), and coniferol (4.003) displayed the highest LE values, whereas aspirin showed an LE value of 2.75. The molecular interactions between the compounds with the best LE values and TLR4 (PDB: 3FXI) are presented in Table S8 and Figure S9. SER-441 was found to form $\mathrm{H}$-bond interactions with phenol and sulcatone, whereas hydrophobic interactions were exhibited for phenol, hydroquinone, catechol, and coniferol. VAL-82 interacted via hydrophobic interactions with coniferol, 4methyl-1,5-heptadiene, catechol, phenol, and hydroquinone. In a previous study, SER-441 was reported to interact strongly with apigenin-7-O-glucoside [87]. Similar interactions were also observed for ILE-80 and VAL-82 [88].

Finally, TRAF6 (PDB: 3HCT) was assessed against the 45 chemicals found in the methanolic extract of $A$. capitiformis stems, of which 34 were identified as interacting (Table 8). Sulcatone (2.47), phenol (2.44), coniferol (2.44), 3-furaldehyde (2.41), 2-(2-hydroxy-2-phenylethyl)-3,5,6-trimethylpyrazine (2.33), and coumaran (2.32) displayed the 


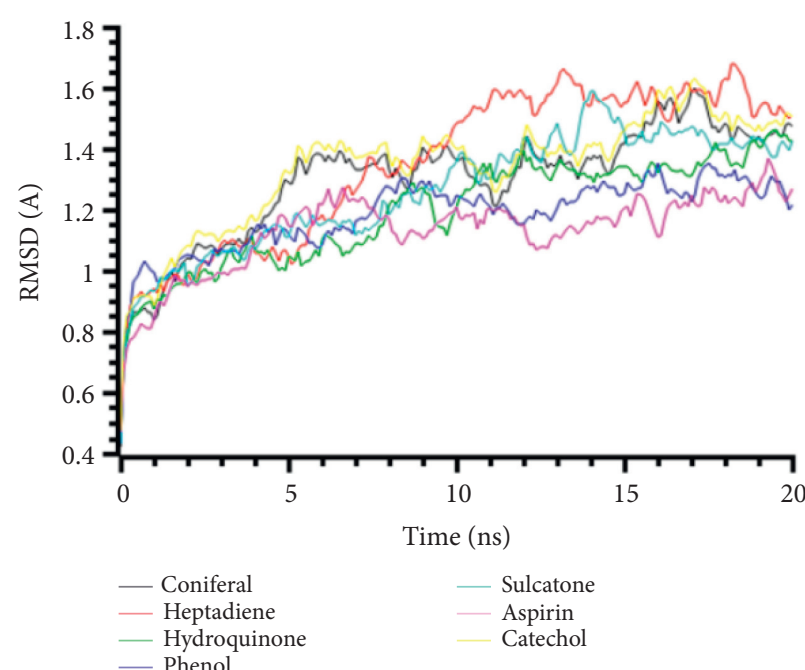

(a)

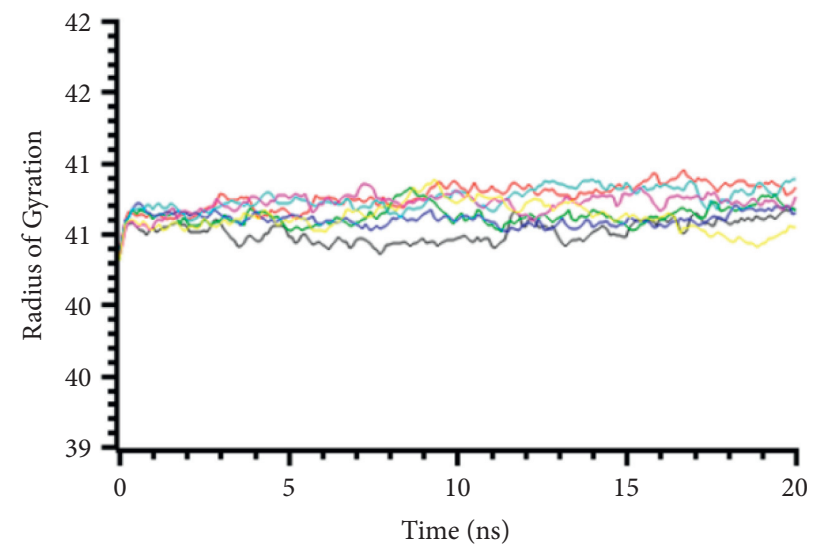

(c)

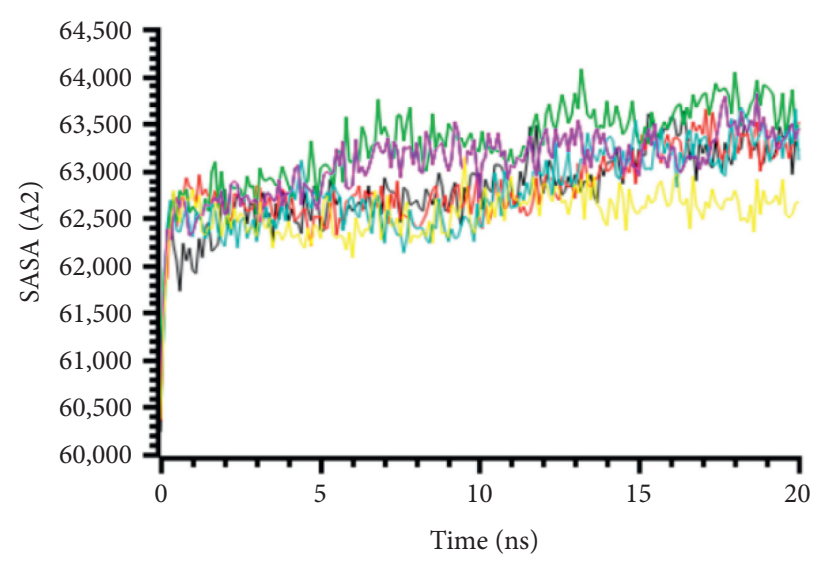

(b)

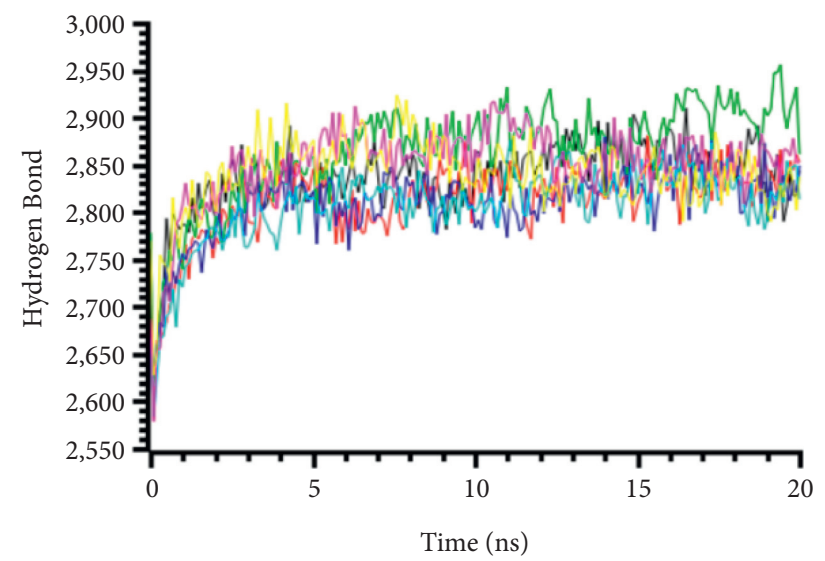

(d)

Figure 4: Two-dimensional representations of the best ligand efficiencies for compounds against TIRAP (PDB: 4FZ5). (a) Root-meansquare deviation of the complexes (RMSD). (b) Solvent accessible surface area (SASA). (c) Radius of gyration (Rg). (d) Hydrogen bond analysis from the simulation system.

highest LE values compared with aspirin (2.07). The molecular interactions between the compounds with the best LE values and TRAF6 (PDB: 3HCT) are presented in Table S9 and Figure S10. ARG-6 formed H-bond interactions with coniferol, 2-(2-hydroxy-2-phenylethyl)-3,5,6trimethylpyrazine, and aspirin, whereas hydrophobic interactions were identified for 2-(2-hydroxy-2-phenylethyl)3,5,6-trimethylpyrazine and sulcatone. GLN-54 also formed $\mathrm{H}$-bond interactions with multiple compounds, including 2-(2-hydroxy-2-phenylethyl)-3,5,6-trimethylpyrazine and 3 -furaldehyde.

The root-mean-square deviations from the C-alpha atoms from the docked complexes IL1R1 (PDB: 1ITB) proteins are illustrated in Figure 1. The complexes' RMSD values were calculated to find out the deviations among the simulation complexes and structural stability. Figure 1(a) demonstrates that the complexes had similar RMSD profiles and did not fluctuate much in the simulation trajectories. The RMSD profile of the complexes reached the steady state after $5 \mathrm{~ns}$ and maintained the structural stability till the last periods of the simulations, which defines the stability of the complexes. The SASA of the complexes was analyzed to find out the change in the surface area. The higher SASA represents the expansion of the surface area of the protein, whereas the lower SASA indicates the truncated nature of the complexes. Figure 1(b) shows that the complexes were in a stable state in SASA. The radius of gyration defines the labiality and mobility of the complexes, where Figure 1(c) indicates the lower deviations. The hydrogen bond patterning follows a similar stable profile (Figure 1(d)).

The molecular dynamics simulation of IRAK4 (PDB: 6EGA) is presented in Figure 2. The RMSD of the complexes had a stable trend in the RMSD profile for all the complexes except catechol. The higher RMSD of these complexes defines the higher flexibility of these compounds in the simulating environments (Figure 2(a)). The SASA profile of the complexes was stable, did not fluctuate much, and had a steady trend in SASA (Figure 2(b)). This SASA profile correlates with the stable and rigid profile of the complexes (Figure 2(c)). The radius of gyration and hydrogen bond 


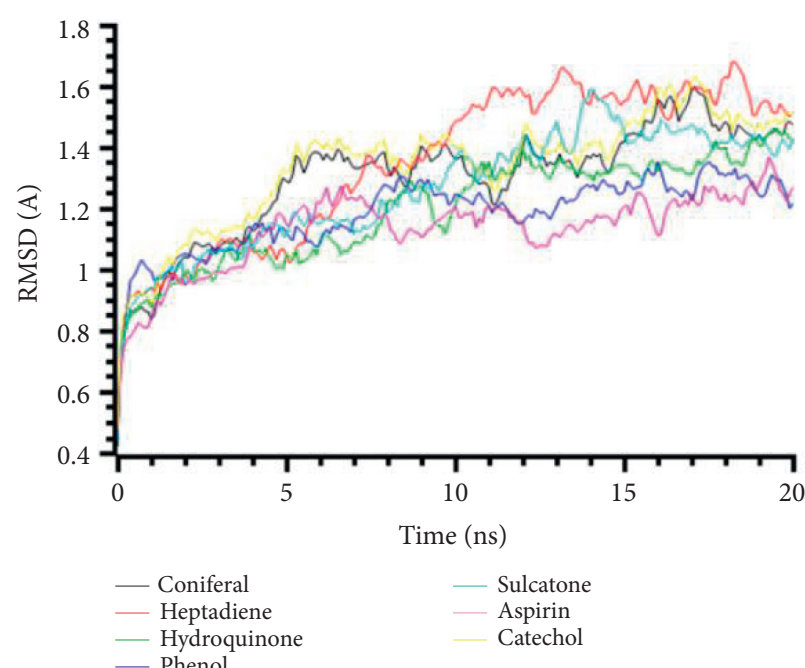

(a)

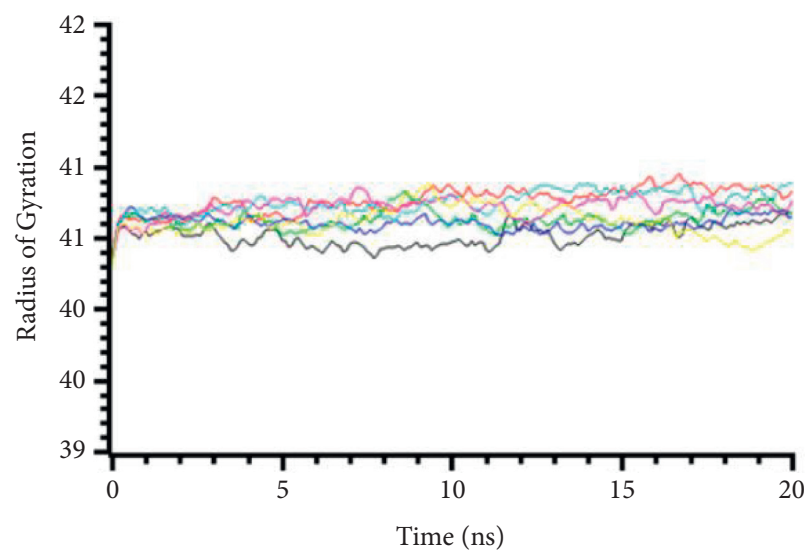

(c)

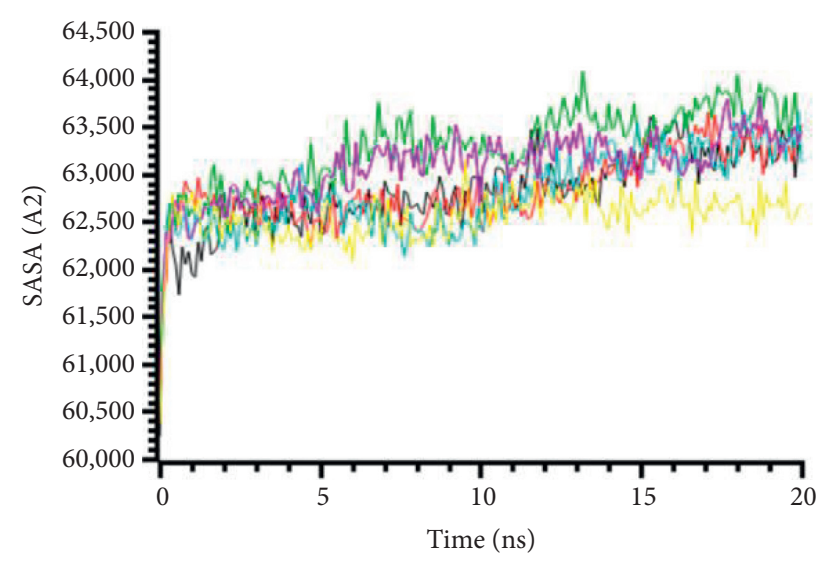

(b)

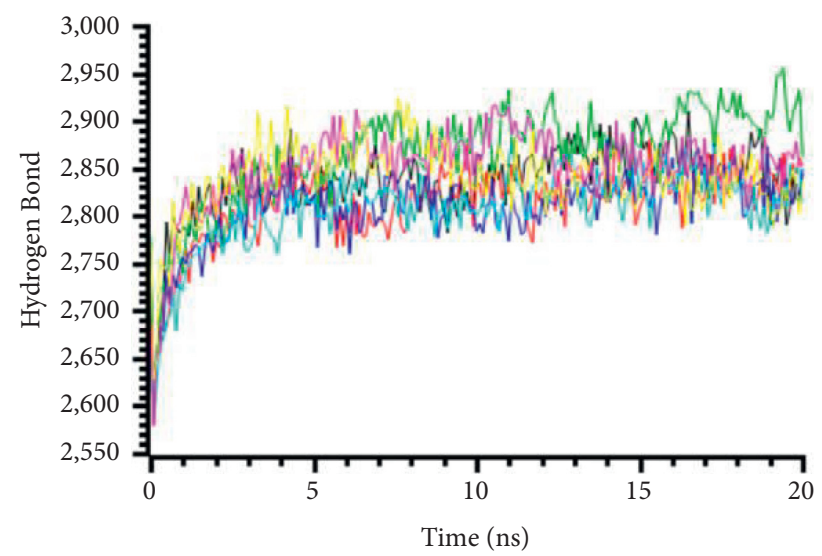

(d)

Figure 5: Two-dimensional representations of the best ligand efficiencies for compounds against TLR4 (PDB: 3FXI). (a) Root-mean-square deviation of the complexes (RMSD). (b) Solvent accessible surface area (SASA). (c) Radius of gyration (Rg). (d) Hydrogen bond analysis from the simulation system.

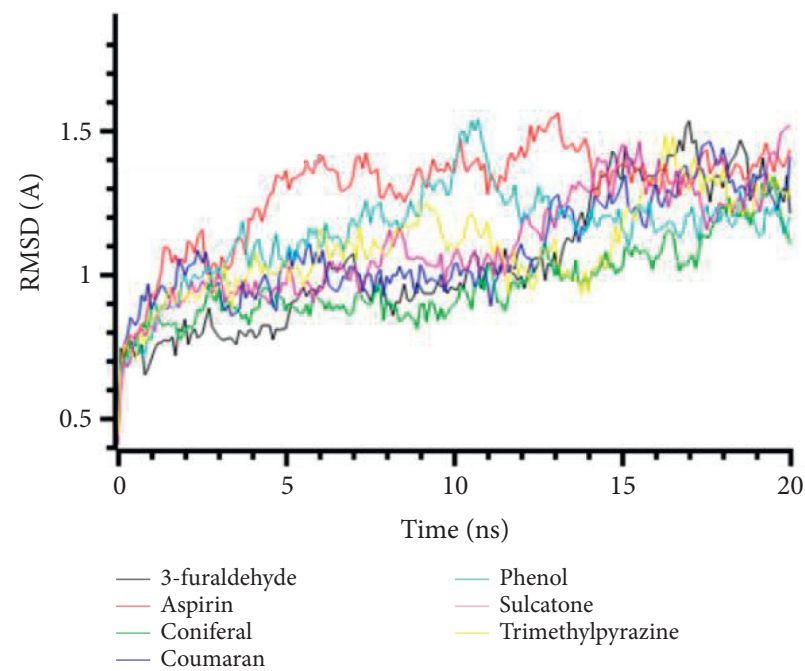

(a)

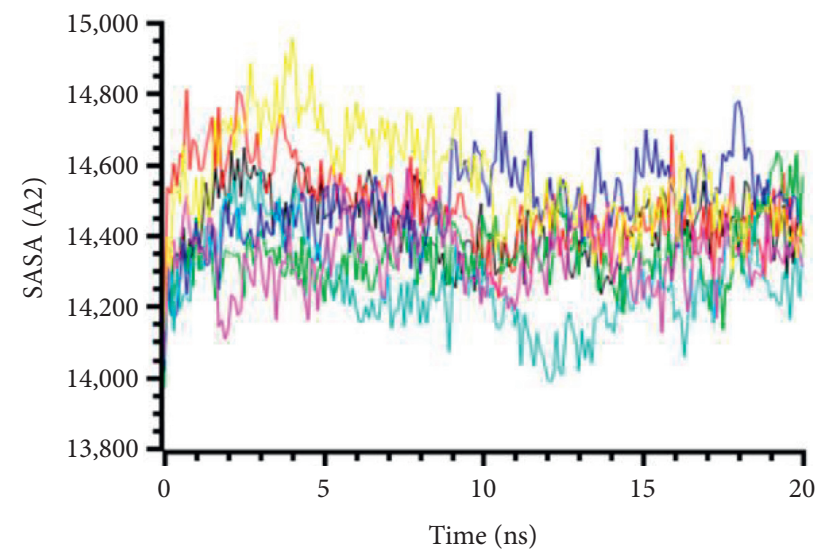

(b)

Figure 6: Continued. 


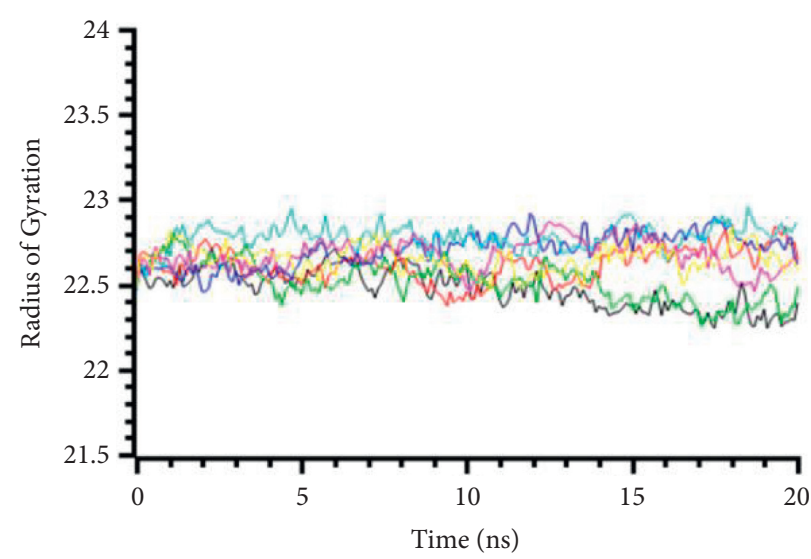

(c)

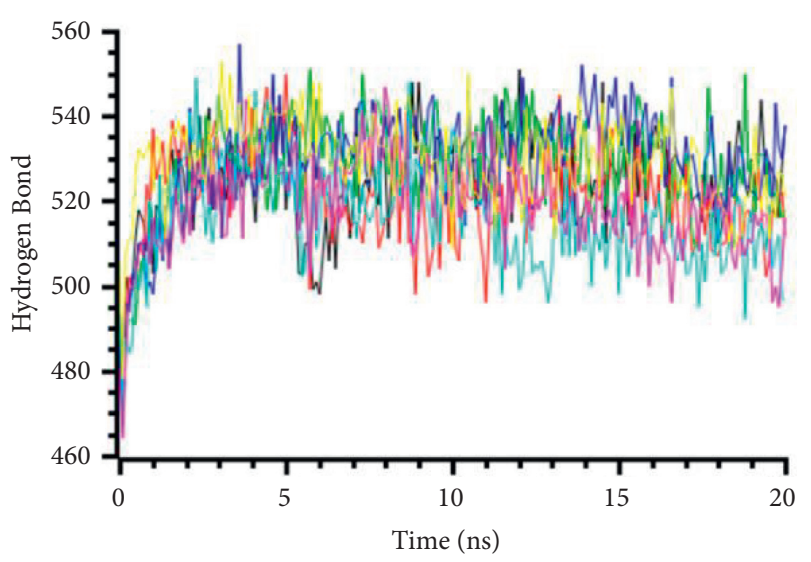

(d)

FiguRE 6: Two-dimensional representations of the best ligand efficiencies for compounds against TRAF6 (PDB: 3HCT). (a) Root-meansquare deviation of the complexes (RMSD). (b) Solvent accessible surface area (SASA). (c) Radius of gyration (Rg). (d) Hydrogen bond analysis from the simulation system.

pattern systems were similar and did not change too much in the simulations (Figure 2(d)).

The RMSD of the MYD88 (4EO7) protein complexes had a lower level of fluctuations, and lower deviations were observed across the compounds. All compounds had a lower RMSD than $2.5 \AA$ in whole simulation periods (Figure 3(a)). The SASA of the MYD88 (4EO7) complexes were stable, but the complex of trans-13docosenamide had a lower SASA than the other complexes. This SASA profile of trans-13-docosenamide defines the MYD88 (4EO7) experienced the condensed conformation upon binding with the corresponding ligands (Figure 3(b)). The 2-(2-Hydroxy-2-phenylethyl)3,5,6-trimethylpyrazine complexes had a higher $\mathrm{Rg}$ than other complexes, which defines the complexes' flexible nature than other complexes (Figure 3(c)). These complexes also had a higher SASA in Figure 3(b), which depicts the changes in the confirmations than other complexes in simulated environments. The hydrogenbonding pattern of the complexes for MYD88 (4EO7) protein was found stable and did not change too much in simulations (Figure 3(d)).

The RMSD profile of TIRAP (PDB: 4FZ5) complexes is illustrated in Figure 4. All complexes from TIRAP (PDB: 4FZ5) protein had an initial rise of RMSD due to a higher degree of flexibility but stabilized subsequently after $5 \mathrm{~ns}$ times. The complex 4-ethylresorcinol had a higher RMSD than the other complexes, which might be responsible for the more remarkable conformational changes and the flexibility of the compounds (Figure 4(a)). The SASA of the complexes had a stable and similar trend for all the compounds. But the complex phenol had a lower SASA profile at the last phase of SASA, which defines the complexes' truncated nature in simulations (Figure 4(b)). The radius of gyration profile of the complexes had a lower trend, which correlates with the less flexibility of the complexes (Figure 4(c)). The hydrogen bond patterning of the complexes had a stable profile in Figure $4(\mathrm{~d})$.
The molecular dynamics simulation study of the TLR4 (PDB: 3FXI) and complexes was done to analyze the structural deviation in the docked structure. The root-meansquare deviations of all complexes are illustrated in Figure 5(a). The RMSD value of the complexes initially followed the upper trend from the beginning. This might be happening due to the higher flexibility level. But all the complexes from TLR4 (PDB: 3FXI) had a stable profile after $10 \mathrm{~ns}$ times and followed a similar trend until the last periods, demonstrating structural stability. The SASA of the TLR4 (PDB: 3FXI) complexes had lowered the degree of the deviations from the beginning and followed lower fluctuations, which define no changes in the surface area of the complexes (Figure 5(b)). The Rg and hydrogen bond patterns of the simulation systems were stable and did not change too much, which correlates with the structural stability (Figures 5(c) and 5(d)).

The docked complexes from the TRAF6 (PDB: 3HCT) protein and their simulation descriptors are illustrated in Figure 6. The RMSD profile of the TRAF6 (PDB: 3HCT) complexes defines that the phenol and aspirin had a higher level of RMSD than other complexes, which correlates with the comparative higher degree of the deviations of the complexes. All complexes had reached a stable state after $5 \mathrm{~ns}$ of the simulation times. Moreover, the complexes exhibit RMSD lower than $2.5 \AA$, which defines the complexes with a higher rigidity degree (Figure 6(a)). The SASA profile of the complexes had a lower deviation, as illustrated in Figure 6(b). The phenol had a lower SASA value than all the compounds, indicating the truncated nature of the protein complexes compared with the others. Moreover, the radius of gyration from Figure 6(c) demonstrates that the complexes had a lower degree of deviations, and no significant higher fluctuations were observed. This $\mathrm{Rg}$ profile indicates the complexes had lower mobility and flexibility during the simulation times. The hydrogen bond pattern of the complexes was stable during the simulations (Figure 6(d)). 


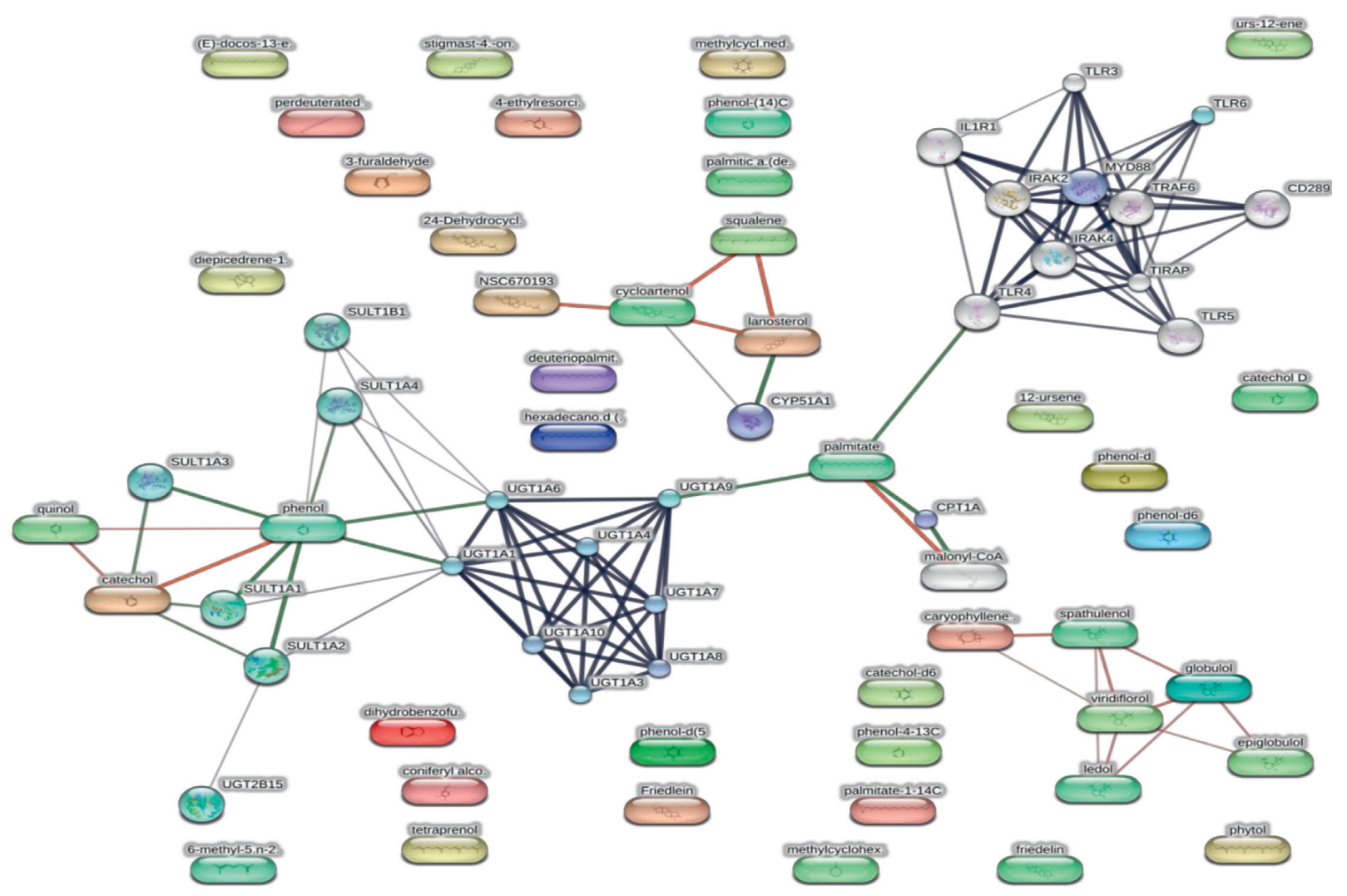

FIGURE 7: Network pharmacology presentation of phytoconstituents, targets, and pathways.

\section{Conclusions}

The network pharmacology analysis revealed key pathways involved in the anti-inflammatory activities induced by the chemical compounds found in the methanolic extract of A. capitiformis stem. Six inflammation pathways were obtained from the KEGG pathway analysis (IL1R1, IRAK4, MYD88, TIRAP, TLR4, and TRAF6), and molecular docking studies of these pathways revealed that the identified chemical compounds had strong binding affinities with these pathway components. The current study discovered that 3-furaldehyde, phenol, catechol, and hydroquinone were effective anti-inflammatory compounds found within the methanolic extract of $A$. capitiformis and played an important part in the inflammation pathway by targeting these six proteins. Additional in vitro and in vivo experiments will be helpful to validate and optimize the findings of this study.

\section{Abbreviations}

GC-MS: Gas chromatography-mass spectrometry

KEGG: $\quad$ Kyoto Encyclopedia of Genes and Genomes

WHO: World Health Organization

NF- $\kappa$ B: $\quad$ Nuclear factor-kappa B

STRING: Search Tool for Retrieval of Interacting Genes

PPIs: $\quad$ Protein-protein interactions

IL: $\quad$ Interleukins
PKC: $\quad$ Protein kinase C

MAPK: Mitogen-activated protein kinase

IRF: Interferon regulatory factor

IRAK: Receptor-associated kinase

TLR: $\quad$ Toll-like receptor

3D: Three-dimensional

PDB: $\quad$ Protein Data Bank

RMSD: Root-mean-square deviation of the complexes

SASA: Solvent accessible surface area

Rg: $\quad$ Radius of gyration.

\section{Data Availability}

The data are available within the manuscript and also accessible from the corresponding author upon request.

\section{Conflicts of Interest}

The authors declare no conflicts of interest.

\section{Authors' Contributions}

Ahmad J. Obaidullah, Mohammed M. Alanazi, Nawaf A. Alsaif, Ashwag S. Alanazi, Hussam Albassam, Osama I. Alwassil, and Alanazi AZ conducted the study and performed the corresponding data analysis, prepared figures, and drafted the manuscript. Abu Montakim Tareq, Mohammed M. Alanazi, and Ahmad J. Obaidullah 
supervised the study, performed the data analysis, prepared the figure, and contributed to writing with Shafi Mahmud and Ali M. Alqahtani. Ahmad J. Obaidullah, Mohammed M. Alanazi, and Abu Montakim Tareq were responsible for writing, review, and editing of the manuscript. All the authors approved the final version of the manuscript.

\section{Acknowledgments}

The authors extend their appreciation to the Deanship of Scientific Research at King Saud University for funding this work through research group no. RG-1441-398.

\section{Supplementary Materials}

Table S1: biological processes of the genes targeted by the compounds; Table S2: molecular functions of the genes interacting with the compounds; Table S3: protein-protein interaction (PPI) status of 10 proteins with coexpression; Table S4: molecular interactions with the best ligand efficiencies for compounds binding with IL1R1 (PDB: 1ITB); Table S5: molecular interactions with the best ligand efficiencies for compounds binding with IRAK4 (PDB: 6EGA); Table S6: molecular interactions with the best ligand efficiencies for compounds binding with MYD88 (4EO7); Table S7: molecular interactions with the best ligand efficiencies for compounds binding with TIRAP (PDB: 4FZ5); Table S8: molecular interactions with the best ligand efficiencies for compounds binding with TLR4 (PDB: 3FXI); and Table S9: molecular interactions with the best ligand efficiencies for compounds binding with TRAF6 (PDB: 3HCT). Figure S1: GC-MS chromatograph of the methanolic extract of Argyreia capitiformis stem; Figure S2: protein-protein interaction (PPI) network of 10 proteins; Figure S3: gene cooccurrence of 10 proteins for Homo sapiens; Figure S4: gene coexpression of 10 proteins in PPI network; and Figure S5: two-dimensional representations of the best ligand efficiencies for compounds binding with IL1R1 (PDB: 1ITB). 3Furaldehyde (A), 4(1H)-pyrimidinone, 6-hydroxy- (B), catechol (C), hydroquinone (D), phenol (E), sulcatone $(\mathrm{F})$, and aspirin $(G)$ are shown. Figure S6: two-dimensional representations of the best ligand efficiencies for compounds binding to IRAK4 (PDB: 6EGA). 3-Furaldehyde (A), 4methyl-1,5-heptadiene (B), catechol (C), methylcyclohexane (D), p-vinylguaiacol (E), phenol (F), and aspirin (G) are shown. Figure S7: two-dimensional representations of the best ligand efficiencies for compounds binding to MYD88 (4EO7). (Z,E)-farnesol (A), 2-(2-hydroxy-2-phenylethyl)3,5,6-trimethylpyrazine (B), 4-methyl-1,5-heptadiene (C), coniferol (D), hydroquinone (E), trans-13-docosenamide $(F)$, and aspirin $(G)$ are shown. Figure S8: two-dimensional representations of the best ligand efficiencies for compounds binding to TIRAP (PDB: 4FZ5). 3-Furaldehyde (A), 4ethylresorcinol (B), catechol (C), hydroquinone (D), methylcyclohexane (E), phenol (F), and aspirin $(G)$ are shown. Figure S9: two-dimensional representations of the best ligand efficiencies for compounds against TLR4 (PDB: 3FXI). 4-Methyl-1,5-heptadiene (A), catechol (B), coniferol
(C), hydroquinone (D), phenol (E), sulcatone (F), and aspirin $(G)$ are shown. Figure S10: two-dimensional representations of the best ligand efficiencies for compounds against TRAF6 (PDB: 3HCT). 2-(2-Hydroxy-2-phenylethyl)-3,5,6-trimethylpyrazine (A), 3-furaldehyde (B), coniferol (C), coumaran (D), phenol (E), sulcatone $(\mathrm{F})$, and aspirin $(\mathrm{G})$ are shown. (Supplementary Materials)

\section{References}

[1] F. O. Martinez, L. Helming, and S. Gordon, "Alternative activation of macrophages: an immunologic functional perspective," Annual Review of Immunology, vol. 27, no. 1, pp. 451-483, 2009.

[2] A. Kauppinen, T. Suuronen, J. Ojala, K. Kaarniranta, and A. Salminen, "Antagonistic crosstalk between NF- $\kappa \mathrm{B}$ and SIRT1 in the regulation of inflammation and metabolic disorders," Cellular Signalling, vol. 25, no. 10, pp. 1939-1948, 2013.

[3] P. Libby, "Inflammatory mechanisms: the molecular basis of inflammation and disease," Nutrition Reviews, vol. 65, no. 12, pp. S140-S146, 2007.

[4] I. P. Barcelos, R. M. Troxell, and J. S. Graves, "Mitochondrial dysfunction and multiple sclerosis," Biology, vol. 8, no. 2, 2019.

[5] D.-H. Tsai, M. Riediker, A. Berchet et al., "Effects of short- and long-term exposures to particulate matter on inflammatory marker levels in the general population," Environmental Science and Pollution Research, vol. 26, no. 19, pp. 19697-19704, 2019.

[6] P. Deepak, J. E. Axelrad, and A. N. Ananthakrishnan, "The role of the radiologist in determining disease severity in inflammatory bowel diseases," Gastrointestinal endoscopy clinics of North America, vol. 29, no. 3, pp. 447-470, 2019.

[7] Y. Zhou, Y. Hong, and H. Huang, "Triptolide attenuates inflammatory response in membranous glomerulo-nephritis rat via downregulation of NF- $\kappa \mathrm{B}$ signaling pathway," Kidney \& Blood Pressure Research, vol. 41, no. 6, pp. 901-910, 2016.

[8] O. Oguntibeju, "Medicinal plants with anti-inflammatory activities from selected countries and regions of Africa," Journal of Inflammation Research, vol. 11, pp. 307-317, 2018.

[9] G. H. Yeum, B. R. So, S. M. Eum, and S. K. Jung, "Evaluation of anti-inflammatory effect by regulating NF- $\kappa$ B pathway of Argyreia capitata (Vahl) Choisy extract in LPS-induced RAW 264.7 macrophages," Korean Journal of Food Science and Technology, vol. 52, no. 3, pp. 249-254, 2020.

[10] H. Singh, P. A. Dhole, R. Saravanan, and P. K. Baske, "Ethnomedicinal plants used in sexual disorder in Balangir and Deogarh districts, Odisha, India," International Journal of Current Science, vol. 20, no. 3, pp. 57-62, 2017.

[11] G.-T. Chen, Y. Lu, M. Yang, J.-L. Li, and B.-Y. Fan, "Medicinal uses, pharmacology, and phytochemistry of Convolvulaceae plants with central nervous system efficacies: a systematic review," Phytotherapy Research, vol. 32, no. 5, pp. 823-864, 2018.

[12] A. H. Atta, N. H. Mohamed, S. M. Nasr, and S. M. Mouneir, "Phytochemical and pharmacological studies on Convolvulus fatmensis Ktze," Journal of Natural Remedies, vol. 7, no. 1, p. 109, 2007.

[13] V. Galani, B. Patel, and N. Patel, "Argyreia speciosa (Linn. f.) sweet: a comprehensive review," Pharmacognosy Reviews, vol. 4, no. 8, pp. 172-178, 2010.

[14] A. Meher, A. Kumar, and P. Ranjan, "A literature review on Argyreia nervosa (burm. F.) bojer," International Journal of 
Research in Ayurveda and Pharmacy, vol. 2, pp. 1501-1504, 2011.

[15] A. B. Gokhale, A. S. Damre, and M. N. Saraf, "Investigations into the immunomodulatory activity of Argyreia speciosa," Journal of Ethnopharmacology, vol. 84, no. 1, pp. 109-114, 2003.

[16] S. Wang, Y. Tong, T. B. Ng et al., "Network pharmacological identification of active compounds and potential actions of Erxian decoction in alleviating menopause-related symptoms," Chinese Medicine, vol. 10, no. 1, pp. 19-12, 2015.

[17] S. Li and B. Zhang, "Traditional Chinese medicine network pharmacology: theory, methodology and application," Chinese Journal of Natural Medicines, vol. 11, no. 2, pp. 110-120, 2013.

[18] Y.-q. Zhang, X. Mao, Q.-y. Guo, N. Lin, and S. Li, "Network pharmacology-based approaches capture essence of Chinese herbal medicines," Chinese Herbal Medicines, vol. 8, no. 2, pp. 107-116, 2016.

[19] J.-w. Liang, M.-y. Wang, K. M. Olounfeh, N. Zhao, S. Wang, and F.-h. Meng, "Network pharmacology-based identification of potential targets of the flower of Trollius chinensis Bunge acting on anti-inflammatory effects," Scientific Reports, vol. 9, no. 1, p. $8109,2019$.

[20] Y. Guo, Q. Nie, A. L. MacLean, Y. Li, J. Lei, and S. Li, "Multiscale modeling of inflammation-induced tumorigenesis reveals competing oncogenic and on coprotective roles for inflammation," Cancer Research, vol. 77, no. 22, pp. 6429-6441, 2017.

[21] T. Yi, S.-M. Li, J.-Y. Fan et al., "Comparative analysis of EPA and DHA in fish oil nutritional capsules by GC-MS," Lipids in Health and Disease, vol. 13, no. 1, p. 190, 2014.

[22] A. M. Juszczak, M. Zovko-Končić, and M. Tomczyk, "Recent trends in the application of chromatographic techniques in the analysis of luteolin and its derivatives," Biomolecules, vol. 9, no. 11, p. 731, 2019.

[23] S. Razack, K. Kumar, I. Nallamuthu, M. Naika, and F. Khanum, "Antioxidant, biomolecule oxidation protective activities of Nardostachys jatamansi DC and its phytochemical analysis by RP-HPLC and GC-MS," Antioxidants, vol. 4, no. 1, pp. 185-203, 2015.

[24] N. Konappa, A. C. Udayashankar, S. Krishnamurthy, C. K. Pradeep, S. Chowdappa, and S. Jogaiah, "GC-MS analysis of phytoconstituents from Amomum nilgiricum and molecular docking interactions of bioactive serverogenin acetate with target proteins," Scientific Reports, vol. 10, no. 1, p. 16438, 2020.

[25] A. M. Tareq, S. Farhad, A. B. M. Neshar Uddin et al., "Chemical profiles, pharmacological properties, and in silico studies provide new insights on Cycas pectinata," Heliyon, vol. 6, no. 6, Article ID e04061, 2020.

[26] M. A. Rahman, R. Sultana, T. Bin Emran et al., "Effects of organic extracts of six Bangladeshi plants on in vitro thrombolysis and cytotoxicity," BMC Complementary and Alternative Medicine, vol. 13, no. 1, p. 25, 2013.

[27] A. J. Obaidullah, M. M. Alanazi, N. A. Alsaif et al., "Deeper insights on Cnesmone javanica blume leaves extract: chemical profiles, biological attributes, network pharmacology and molecular docking," Plants, vol. 10, no. 4, 2021.

[28] P. Khanal, B. M. Patil, B. K. Mandar, Y. N. Dey, and T. Duyu, "Network pharmacology-based assessment to elucidate the molecular mechanism of anti-diabetic action of Tinospora cordifolia," Clinical Phytoscience, vol. 5, no. 1, pp. 1-9, 2019.

[29] M. F. Mahomoodally, S. Jugreet, K. I. Sinan et al., "Pharmacological potential and chemical characterization of bridelia ferruginea benth-a native tropical african medicinal plant," Antibiotics, vol. 10, no. 2, p. 223, 2021.

[30] D. Szklarczyk, A. Franceschini, S. Wyder et al., "STRING v10: protein-protein interaction networks, integrated over the tree of life," Nucleic Acids Research, vol. 43, pp. D447-D452, 2015.

[31] G. P. A. Vigers, L. J. Anderson, P. Caffes, and B. J. Brandhuber, "Crystal structure of the type-I interleukin-1 receptor complexed with interleukin-1 $\beta$," Nature, vol. 386, no. 6621, pp. 190-194, 1997.

[32] L. Wang, R. Ferrao, Q. Li et al., "Conformational flexibility and inhibitor binding to unphosphorylated interleukin-1 receptor-associated kinase 4 (IRAK4)," Journal of Biological Chemistry, vol. 294, no. 12, pp. 4511-4519, 2019.

[33] G. A. Snyder, C. Cirl, J. Jiang et al., "Molecular mechanisms for the subversion of MyD88 signaling by TcpC from virulent uropathogenic Escherichia coli," Proceedings of the National Academy of Sciences, vol. 110, no. 17, pp. 6985-6990, 2013.

[34] J.-R. Woo, S.-M. Kim, S. E. Shoelson, and S.-Y. Park, "X-ray crystallographic structure of TIR-domain from the human TIR-domain containing adaptor protein/MyD88-adaptor-like protein (TIRAP/MAL)," Bulletin of the Korean Chemical Society, vol. 33, no. 9, pp. 3091-3094, 2012.

[35] B. S. Park, D. H. Song, H. M. Kim, B.-S. Choi, H. Lee, and J.-O. Lee, "The structural basis of lipopolysaccharide recognition by the TLR4-MD-2 complex," Nature, vol. 458, no. 7242, pp. 1191-1195, 2009.

[36] Q. Yin, S.-C. Lin, B. Lamothe et al., "E2 interaction and dimerization in the crystal structure of TRAF6," Nature Structural \& Molecular Biology, vol. 16, no. 6, pp. 658-666, 2009.

[37] E. Lionta, G. Spyrou, D. Vassilatis, and Z. Cournia, "Structure-based virtual screening for drug discovery: principles, applications and recent advances," Current Topics in Medicinal Chemistry, vol. 14, no. 16, pp. 1923-1938, 2014.

[38] F. Chen, H. Liu, H. Sun et al., "Assessing the performance of the MM/PBSA and MM/GBSA methods. 6. Capability to predict protein-protein binding free energies and re-rank binding poses generated by protein-protein docking," Physical Chemistry Chemical Physics, vol. 18, no. 32, pp. 22129-22139, 2016.

[39] A. T. García-Sosa, C. Hetényi, and U. Maran, "Drug efficiency indices for improvement of molecular docking scoring functions," Journal of Computational Chemistry, vol. 31, no. 1, pp. 174-184, 2010.

[40] J. Wang, R. M. Wolf, J. W. Caldwell, P. A. Kollman, and D. A. Case, "Development and testing of a general amber force field," Journal of Computational Chemistry, vol. 25, no. 9, pp. 1157-1174, 2004.

[41] H. Land and M. S. Humble, "YASARA: a tool to obtain structural guidance in biocatalytic investigations," Methods in Molecular Biology, vol. 1685, pp. 43-67, 2018.

[42] M. F. Harrach and B. Drossel, "Structure and dynamics of TIP3P, TIP4P, and TIP5P water near smooth and atomistic walls of different hydroaffinity," The Journal of Chemical Physics, vol. 140, no. 17, Article ID 174501, 2014.

[43] M. J. Harvey and G. De Fabritiis, "An implementation of the smooth particle mesh ewald method on GPU hardware," Journal of Chemical Theory and Computation, vol. 5, no. 9, pp. 2371-2377, 2009.

[44] U. Essmann, L. Perera, M. L. Berkowitz, T. Darden, H. Lee, and L. G. Pedersen, "A smooth particle mesh Ewald method," The Journal of Chemical Physics, vol. 103, no. 19, pp. 8577-8593, 1995. 
[45] E. Krieger and G. Vriend, "New ways to boost molecular dynamics simulations," Journal of Computational Chemistry, vol. 36, no. 13, pp. 996-1007, 2015.

[46] S. Mahmud, M. A. R. Uddin, G. K. Paul et al., "Virtual screening and molecular dynamics simulation study of plantderived compounds to identify potential inhibitors of main protease from SARS-CoV-2," Briefings in Bioinformatics, vol. 22, no. 2, pp. 1402-1414, 2021.

[47] S. S. Bappy, S. Sultana, J. Adhikari et al., "Extensive immunoinformatics study for the prediction of novel peptide-based epitope vaccine with docking confirmation against envelope protein of Chikungunya virus: a computational biology approach," Journal of Biomolecular Structure and Dynamics, vol. 39, no. 4, pp. 1139-1154, 2021.

[48] M. A. Khan, S. Mahmud, A. S. M. R. U. Alam, M. E. Rahman, F. Ahmed, and M. Rahmatullah, "Comparative molecular investigation of the potential inhibitors against SARS-CoV-2 main protease: a molecular docking study," Journal of biomolecular structure \& dynamics, vol. 39, no. 16, pp. 6317-6323, 2021.

[49] S. K. Pramanik, S. Mahmud, G. K. Paul et al., "Fermentation optimization of cellulase production from sugarcane bagasse by Bacillus pseudomycoides and molecular modeling study of cellulase," Current Research in Microbial Sciences, vol. 2, Article ID 100013, 2021.

[50] K. H. Chowdhury, M. R. Chowdhury, S. Mahmud et al., "Drug repurposing approach against novel coronavirus disease (COVID-19) through virtual screening targeting SARS-CoV2 main protease," Biology, vol. 10, no. 1, 2020.

[51] M. Munia, S. Mahmud, M. Mohasin, and K. M. K. Kibria, "In silico design of an epitope-based vaccine against choline binding protein A of Streptococcus pneumoniae," Informatics in Medicine Unlocked, vol. 23, Article ID 100546, 2021.

[52] A. Rakib, Z. Nain, S. A. Sami et al., "A molecular modelling approach for identifying antiviral selenium-containing heterocyclic compounds that inhibit the main protease of SARSCoV-2: an in silico investigation," Briefings in Bioinformatics, vol. 22, no. 2, pp. 1476-1498, 2021.

[53] S. Mahmud, M. A. R. Uddin, M. Zaman et al., "Molecular docking and dynamics study of natural compound for potential inhibition of main protease of SARS-CoV-2," Journal of Biomolecular Structure \& Dynamics, vol. 39, no. 16, pp. 6281-6289, 2020.

[54] M. Z. Uddin, A. Paul, A. Rakib et al., "Chemical profiles and pharmacological properties with in silico studies on elatostema papillosum wedd," Molecules, vol. 26, no. 4, p. 809, 2021.

[55] B. Petrovska, "Historical review of medicinal plants' usage," Pharmacognosy Reviews, vol. 6, no. 11, pp. 1-5, 2012.

[56] I. Ahmad, F. Aqil, and M. Owais, Modern Phytomedicine: Turning Medical Plants into Drugs, Wiley VCH, Weinheim, Germany, 2006.

[57] K. Nisha, M. Darshana, G. Madhu, and M. K. Bhupendra, "GC-MS analysis and anti-microbial activity of Psidium guajava (leaves) grown in Malva region of India," International Journal of Drug Development \& Research, vol. 3, no. 4, pp. 237-245, 2011.

[58] K. Gopalakrishnan and R. Udayakumar, "GC-MS analysis of phytocompounds of leaf and stem of Marsilea quadrifolia (L.)," International Journal of Biochemistry Research \& Review, vol. 4, no. 6, pp. 517-526, 2014.

[59] T. Starlin, P. S. Prabha, P. Saravana Prabha, B. K. A. Thayakumar, and V. K. Gopalakrishnan, "Screening and GC-MS profiling of ethanolic extract of Tylophora pauciflora," Bioinformation, vol. 15, no. 6, pp. 425-429, 2019.
[60] T. Efferth, P. C. H. Li, V. S. B. Konkimalla, and B. Kaina, "From traditional Chinese medicine to rational cancer therapy," Trends in Molecular Medicine, vol. 13, no. 8, pp. 353-361, 2007.

[61] K. Ghazi-Moghadam, H. M. Inançl, N. Bazazy, P. K. Plinkert, T. Efferth, and S. Sertel, "Phytomedicine in otorhinolaryngology and pulmonology: clinical trials with herbal remedies," Pharmaceuticals, vol. 5, no. 8, pp. 853-874, 2012.

[62] D. J. Loegering and M. R. Lennartz, "Protein kinase C and tolllike receptor signaling," Enzyme Research, vol. 2011, Article ID 537821, 7 pages, 2011.

[63] D. Szklarczyk, A. L. Gable, D. Lyon et al., "STRING v11: protein-protein association networks with increased coverage, supporting functional discovery in genome-wide experimental datasets," Nucleic Acids Research, vol. 47, no. D1, pp. D607-D613, 2019.

[64] L. Xie and P. E. Bourne, "Functional coverage of the human genome by existing structures, structural genomics targets, and homology models," PLoS Computational Biology, vol. 1, no. 3, p. e31, 2005.

[65] M. Uhlen, P. Oksvold, L. Fagerberg et al., "Towards a knowledge-based human protein atlas," Nature Biotechnology, vol. 28, no. 12, pp. 1248-1250, 2010.

[66] "UniProt: The universal protein knowledgebase," Nucleic Acids Research, vol. 45, no. D1, pp. D158-D169, 2017.

[67] N. J. Gay and M. Gangloff, "Structure and function of Toll receptors and their ligands," Annual Review of Biochemistry, vol. 76, no. 1, pp. 141-165, 2007.

[68] T. Kawai and S. Akira, "Signaling to NF- $\kappa$ B by toll-like receptors," Trends in Molecular Medicine, vol. 13, no. 11, pp. 460-469, 2007.

[69] T. Kawai and S. Akira, "The role of pattern-recognition receptors in innate immunity: update on Toll-like receptors," Nature Immunology, vol. 11, no. 5, pp. 373-384, 2010.

[70] M. Huynen, B. Snel, W. Lathe III, and P. Bork, "Predicting protein function by genomic context: quantitative evaluation and qualitative inferences," Genome Research, vol. 10, no. 8, pp. 1204-1210, 2000.

[71] L. Skrabanek, H. K. Saini, G. D. Bader, and A. J. Enright, "Computational prediction of protein-protein interactions," Molecular Biotechnology, vol. 38, no. 1, pp. 1-17, 2008.

[72] T. Barrett, S. E. Wilhite, P. Ledoux et al., "NCBI GEO: archive for functional genomics data sets-update," Nucleic Acids Research, vol. 41, pp. D991-D995, 2013.

[73] A. Lavecchia and C. Giovanni, "Virtual screening strategies in drug discovery: a critical review," Current Medicinal Chemistry, vol. 20, no. 23, pp. 2839-2860, 2013.

[74] T. Cheng, Q. Li, Z. Zhou, Y. Wang, and S. H. Bryant, "Structure-based virtual screening for drug discovery: a problem-centric review," The AAPS Journal, vol. 14, no. 1, pp. 133-141, 2012.

[75] C. A. Dinarello, "Immunological and inflammatory functions of the interleukin-1 family," Annual Review of Immunology, vol. 27, no. 1, pp. 519-550, 2009.

[76] P. Martin, G. Palmer, S. Vigne et al., "Mouse neutrophils express the decoy type 2 interleukin-1 receptor (IL-1R2) constitutively and in acute inflammatory conditions," Journal of Leukocyte Biology, vol. 94, no. 4, pp. 791-802, 2013.

[77] K. Shimizu, A. Nakajima, K. Sudo et al., "IL-1 receptor type 2 suppresses collagen-induced arthritis by inhibiting IL-1 signal on macrophages," The Journal of Immunology, vol. 194, no. 7, pp. 3156-3168, 2015.

[78] C. Brikos, R. Wait, S. Begum, L. A. J. O’Neill, and J. Saklatvala, "Mass spectrometric analysis of the endogenous type I 
interleukin-1 (IL-1) receptor signaling complex formed after IL-1 binding identifies IL-1RAcP, MyD88, and IRAK-4 as the stable components," Molecular \& Cellular Proteomics, vol. 6, no. 9, pp. 1551-1559, 2007.

[79] R. Sarabu, J. P. Cooper, C. M. Cook, P. Gillespie, A. V. Perrotta, and G. L. Olson, "Design and synthesis of small molecule interleukin-1 receptor antagonists based on a benzene template," Drug Design and Discovery, vol. 15, no. 3, pp. 191-198, 1998.

[80] L. A. J. O’Neill, “The interleukin-1 receptor/Toll-like receptor superfamily: 10 years of progress," Immunological Reviews, vol. 226, no. 1, pp. 10-18, 2008.

[81] S. Akira, S. Uematsu, and O. Takeuchi, "Pathogen recognition and innate immunity," Cell, vol. 124, no. 4, pp. 783-801, 2006.

[82] K. A. Fitzgerald, E. M. Palsson-McDermott, A. G. Bowie et al., "Mal (MyD88-adapter-like) is required for toll-like receptor-4 signal transduction," Nature, vol. 413, no. 6851, pp. 78-83, 2001.

[83] T. Horng, G. M. Barton, and R. Medzhitov, "TIRAP: an adapter molecule in the Toll signaling pathway," Nature Immunology, vol. 2, no. 9, pp. 835-841, 2001.

[84] T. Horng, G. M. Barton, R. A. Flavell, and R. Medzhitov, "The adaptor molecule TIRAP provides signalling specificity for toll-like receptors," Nature, vol. 420, no. 6913, pp. 329-333, 2002.

[85] M. Yamamoto, S. Sato, H. Hemmi et al., "Essential role for TIRAP in activation of the signalling cascade shared by TLR2 and TLR4," Nature, vol. 420, no. 6913, pp. 324-329, 2002.

[86] O. Kadioglu, J. Nass, M. E. Saeed, B. Schuler, and T. Efferth, "Kaempferol is an anti-inflammatory compound with activity towards NF- $\kappa \mathrm{B}$ pathway proteins," Anticancer Research, vol. 35, no. 5, pp. 2645-2650, 2015.

[87] W. A. H. M. Karunarathne, I. M. N. Molagoda, K. T. Lee et al., "Anthocyanins from Hibiscus syriacus L. attenuate LPS-induced inflammation by inhibiting the TLR4-mediated NF- $\kappa \mathrm{B}$ signaling pathway," in Proceedings of the Plant Resources Society of Korea Conference, p. 92, Seoul, Korea, November 2019.

[88] Q. U. Ain, M. Batool, and S. Choi, "TLR4-Targeting therapeutics: structural basis and computer-aided drug discovery approaches," Molecules, vol. 25, no. 3, p. 627, 2020. 